Review

\title{
Systematic Review of Mind-Body Interventions to Treat Myalgic Encephalomyelitis/Chronic Fatigue Syndrome
}

\author{
Samaneh Khanpour Ardestani ${ }^{1}$, Mohammad Karkhaneh ${ }^{2}$, Eleanor Stein ${ }^{3}$, Salima Punja ${ }^{1}$, Daniela R. Junqueira ${ }^{1}$, \\ Tatiana Kuzmyn ${ }^{4}$, Michelle Pearson ${ }^{5}$, Laurie Smith ${ }^{6}$, Karin Olson ${ }^{7}$ and Sunita Vohra ${ }^{8, *}$ \\ 1 Department of Pediatrics, Faculty of Medicine \& Dentistry, University of Alberta, \\ Edmonton, AB T6G 1C9, Canada; khanpour@ualberta.ca (S.K.A.); punja@ualberta.ca (S.P.); \\ junqueir@ualberta.ca (D.R.J.) \\ 2 Institute of Health Economics, Edmonton, AB T6X 0E1, Canada; mk4@ualberta.ca \\ 3 Department of Psychiatry, Faculty of Medicine, University of Calgary, Calgary, AB T2T4L8, Canada; \\ espc@eleanorsteinmd.ca \\ 4 Patient Research Partner, Retired RN, Patient and Community Engagement Research (PaCER) Program \\ Graduate, University of Calgary, Calgary, AB T2P 1B2, Canada; tkuzmyn@telus.net \\ 5 Patient Research Partner, MAPC, CEO Wunjo IS, Calgary, AB T3K 4N8, Canada; michelle@wunjo-is.com \\ 6 Patient Research Partner, Calgary, AB 95060, Canada; lauriem.smith@gmail.com \\ 7 Faculty of Nursing, University of Alberta, Edmonton, AB T6G 1C9, Canada; karin.olson@ualberta.ca \\ 8 Departments of Pediatrics and Psychiatry, Faculty of Medicine \& Dentistry, University of Alberta, \\ Edmonton, AB T6G 1C9, Canada \\ * Correspondence: svohra@ualberta.ca; Tel.: +1-780-492-6445
}

\section{check for} updates

Citation: Khanpour Ardestani, S.; Karkhaneh, M.; Stein, E.; Punja, S.; Junqueira, D.R.; Kuzmyn, T.; Pearson, M.; Smith, L.; Olson, K.; Vohra, S. Systematic Review of Mind-Body Interventions to Treat Myalgic Encephalomyelitis/Chronic Fatigue Syndrome. Medicina 2021, 57, 652. https://doi.org/10.3390/

medicina57070652

Academic Editor: Derek F. H. Pheby

Received: 11 May 2021

Accepted: 17 June 2021

Published: 24 June 2021

Publisher's Note: MDPI stays neutral with regard to jurisdictional claims in published maps and institutional affiliations.

Copyright: (C) 2021 by the authors Licensee MDPI, Basel, Switzerland. This article is an open access article distributed under the terms and conditions of the Creative Commons Attribution (CC BY) license (https:/ / creativecommons.org/licenses/by/ $4.0 /)$.

\begin{abstract}
Background and Objectives: Myalgic Encephalomyelitis/Chronic Fatigue Syndrome (ME/CFS) is a chronic condition distinguished by disabling fatigue associated with post-exertional malaise, as well as changes to sleep, autonomic functioning, and cognition. Mind-body interventions (MBIs) utilize the ongoing interaction between the mind and body to improve health and wellbeing. Purpose: To systematically review studies using MBIs for the treatment of ME/CFS symptoms. Materials and Methods: MEDLINE, EMBASE, CINAHL, PsycINFO, and Cochrane CENTRAL were searched (inception to September 2020). Interventional studies on adults diagnosed with ME/CFS, using one of the MBIs in comparison with any placebo, standard of care treatment or waitlist control, and measuring outcomes relevant to the signs and symptoms of ME/CFS and quality of life were assessed for inclusion. Characteristics and findings of the included studies were summarized using a descriptive approach. Results: 12 out of 382 retrieved references were included. Seven studies were randomized controlled trials (RCTs) with one including three reports (1 RCT, 2 singlearms); others were single-arm trials. Interventions included mindfulness-based stress reduction, mindfulness-based cognitive therapy, relaxation, Qigong, cognitive-behavioral stress management, acceptance and commitment therapy and isometric yoga. The outcomes measured most often were fatigue severity, anxiety/depression, and quality of life. Fatigue severity and symptoms of anxiety/depression were improved in nine and eight studies respectively, and three studies found that MBIs improved quality of life. Conclusions: Fatigue severity, anxiety/depression and physical and mental functioning were shown to be improved in patients receiving MBIs. However, small sample sizes, heterogeneous diagnostic criteria, and a high risk of bias may challenge this result. Further research using standardized outcomes would help advance the field.
\end{abstract}

Keywords: myalgic encephalomyelitis/chronic fatigue syndrome; mind-body interventions; systematic review; adults

\section{Introduction}

Myalgic encephalomyelitis/chronic fatigue syndrome (ME/CFS) is a chronic condition distinguished by disabling fatigue associated with multiple symptoms including postexertional malaise, orthostatic intolerance, pain, sleep problems, and impaired cognitive 
and immune functions [1]. While the true prevalence is unknown, Johnston et al., estimated the pooled prevalence of ME/CFS to be $3.28 \%$ and $0.76 \%$ according to self-reporting and clinical assessment, respectively [2]. In Canada, $1.4 \%$ of people older than 12 years old [3] suffer from ME/CFS. Patients report post-exertional malaise (69-100\%), muscle pain (63-95\%), impaired memory or concentration (88\%), non-restorative sleep $(87 \%)$, joint pain $(55-85 \%)$, and sore throat $(62 \%)[1,4]$. Health-related quality of life in ME/CFS patients is consistently reported as significantly lower than otherwise healthy populations with regards to physical and mental health, self-care, and ability to perform usual activities [5,6]. Not surprisingly, ME/CFS reduces patients' abilities to carry out normal working activities leading to higher unemployment rates [7]. It is estimated that annual household and labor force productivity of ME/CFS patients are decreased by $37 \%$ and $54 \%$, respectively, costing an approximate annual loss of $\$ 9.1$ billion in the United States (US) [8]. ME/CFS patients, their families and employers endure a high financial burden estimated to be between $\$ 18$ to $\$ 51$ billion annually in the US [9].

Despite extensive research, the etiology and pathophysiology of ME/CFS have not yet been fully understood. Disruptions in the autonomic nervous system, hypothalamicpituitary-adrenocortical (HPA) axis, and immune system were shown in several studies [10,11]. Metabolic and mitochondrial dysfunction and abnormal gut microbiota were also shown to be interconnected with the above dysregulation [11]. A recent systematic review of neuroimaging studies showed inconsistent but widespread abnormalities in white matter, functional connectivity, and morphological changes of the autonomic nervous system [12].

With no specific etiology, there is no gold standard method to diagnose ME/CFS to date. A recent systematic review of diagnostic methods by Haney et al., identified nine case definitions [13]. Due to the lack of a biomarker, most of the case definitions require other competing diagnoses to be ruled out $[14,15]$. In the literature, the term myalgic encephalomyelitis (ME) [16] was used earlier than the term chronic fatigue syndrome (CFS) [17]. The Canadian case definition published in 2003 required post-exertional malaise as an essential symptom in these patients and recommended the umbrella term $\mathrm{ME} / \mathrm{CFS}$ [18], used in this systematic review.

There is no cure for ME/CFS nor any FDA or Health Canada approved medication to treat it $[14,19]$, therefore the focus tends to be on managing and minimizing the symptoms and improving quality of life. A variety of conventional and complementary therapies have been used to mitigate the symptoms of ME/CFS. As in other chronic conditions, long-term pharmacological interventions may have significant impacts on patients and their families in terms of adverse effects and financial burden [20,21]. Non-pharmacological options are of interest to patients as they may be less expensive and have fewer associated adverse effects.

Systematic reviews have shown low strength of evidence for the effectiveness of different complementary therapies [19], cognitive-behavioral therapy (CBT), counseling and behavioral therapies [14,22], and graded exercise therapy [23] for improvement of fatigue, physical functioning, sleep, and quality of life in patients with ME/CFS.

Mind-body approaches utilize the interactions between the brain, mind, and body, and behavior to improve health and wellbeing [24]. Using these interconnections strengthens self-awareness and self-care and helps to improve mood, quality of life, and increase one's ability to cope. Examples of mind-body therapy interventions (MBIs) include progressive muscle relaxation, guided imagery, hypnosis, meditation, mindfulness, Tai chi, yoga, and biofeedback. Newer approaches are using the brain's ability to change (i.e., neuroplasticity) associated with repeated, purposeful thoughts, feelings or behaviors [25]. The science behind how mind-body therapies work is expanding. It has been shown that the brain and body communicate in multiple directions using neurotransmitters/neuropeptides, hormones, and cytokines and MBIs may be influencing physical health by affecting these interactions [24,26].

Considering the complex nature of ME/CFS and the involvement of psycho-neuroendocrine and immune systems, these patients are an ideal population for evaluating MBIs. Further- 
more, by enhancing self-knowledge and patients' abilities to work through their problems and reduce stress, MBIs may improve their quality of life and wellbeing [27].

Several MBIs such as mindfulness-based stress reduction (MBSR), mindfulness-based cognitive therapy (MBCT), yoga, and Qigong have been studied in ME/CFS patients, but to our knowledge, have not yet been included in any systematic review or meta-analysis. There are some promising results to improve anxiety, fatigue, depression, quality of life, and physical functioning [28-32] in ME/CFS. In this systematic review, we evaluated the effectiveness and safety of MBIs that were studied in individuals diagnosed with ME/CFS. The results of this review will inform the design and methodology of future randomized controlled trials.

\section{Objectives}

The objectives of this study were to systematically review studies of MBIs for the treatment of ME/CFS symptoms and to report any adverse events reported for these approaches in ME/CFS patients.

\section{Materials and Methods}

We followed the Preferred Reporting Items for Systematic Reviews and Meta-Analysis (PRISMA) guidelines [33]. The protocol of this systematic review was registered at PROSPERO (CRD42018085981).

\subsection{Population, Intervention, Control, Outcome- Study Design (PICO-S)}

The population of interest was adults ( $\geq 18$ years old) diagnosed or symptom-matched with one of the ME/CFS case definitions (Appendix A, Table A1). Patients with any other conditions were included in this review, as long as they were diagnosed with ME/CFS. Interventions of interest included any of the MBIs listed in Table 1 and any placebo, the standard of care treatment or waiting list as a control group. To be eligible for inclusion, multiple-arm interventional studies were also required to have at least one of the control groups mentioned above.

All outcomes relevant to the signs and symptoms of ME/CFS and quality of life were considered. The outcomes included fatigue, sleep refreshment, pain, anxiety (stress, nervousness, etc.), depression (mood, hopefulness, and helplessness), quality of life, performance (physical, mental, emotional), work-related outcomes (employment, income, etc.), and physical health symptoms such as sore throat, tender lymph nodes, and muscle weakness (Table 1 ).

Study designs eligible for inclusion were parallel/cross-over/N-of-1 randomized controlled trials (RCTs), controlled clinical trials (CCTs), single-arm experimental (within subject control group), controlled before and after studies, or cohort studies.

\subsection{Search Methods}

Five electronic databases (MEDLINE, EMBASE, CINAHL, PsycINFO, and Cochrane Register of Controlled Trials (CENTRAL)) were searched from inception to September 2020. Search terms were based on those presented in Table 1; an example is found in Appendix B. No limitation was implemented in terms of publication dates. English language restriction was applied. The reference lists of included studies, and systematic reviews, were reviewed to identify additional studies. 
Table 1. Criteria for selecting studies.

\begin{tabular}{|c|c|}
\hline Population & $\begin{array}{l}\text { Patients with a diagnosis of CFS, ME, and ME/CFS including: } \\
\text { Patients who were previously treated } \\
\text { Patients who are previously untreated } \\
\text { Adults ( } \geq 18 \text { years) }\end{array}$ \\
\hline Interventions & $\begin{array}{l}\text { Mind-body interventions (alone or in combination) including: } \\
\text { Art Therapy } \\
\text { Autogenic training } \\
\text { Biofeedback/neurofeedback } \\
\text { Breathing exercise } \\
\text { Cognitive restructuring } \\
\text { Dynamic Neural Retraining System } \\
\text { Emotional Freedom Techniques (EFT) } \\
\text { Eye movement desensitization and reprocessing (EMDR) } \\
\text { Guided imagery } \\
\text { Hypnotherapy/self-hypnosis } \\
\text { Meditation (mindfulness, mantra, guided, transcendental) } \\
\text { Mindfulness-based cognitive therapy (MBCT) } \\
\text { Mindfulness-based Stress Reduction (MBSR) } \\
\text { Music therapy } \\
\text { Neurolinguistic programming } \\
\text { Prayer/spirituality } \\
\text { Psychological flexibility } \\
\text { Qigong } \\
\text { Relaxation therapy (relaxation response, progressive muscle relaxation) } \\
\text { Tai Chi } \\
\text { Visualization } \\
\text { Yoga }\end{array}$ \\
\hline Comparators & $\begin{array}{l}\text { One or more of the following control conditions including: } \\
\text { Placebo } \\
\text { Standard of care treatments } \\
\text { Waitlist }\end{array}$ \\
\hline Outcomes & $\begin{array}{l}\text { Any single or combination of, but not limited to, the following outcomes: } \\
\text { Fatigue (energy, motivation) } \\
\text { Refreshing sleep } \\
\text { Pain } \\
\text { Anxiety (stress, nervousness, etc.,) } \\
\text { Depression (mood, hopefulness, helplessness) } \\
\text { Quality of life } \\
\text { Performance (physical, mental, emotional) } \\
\text { Work-related outcome (employment, income, etc.) } \\
\text { Changes in physical health such as sore throat, tender lymph nodes, and muscle weakness }\end{array}$ \\
\hline Study Design & $\begin{array}{l}\text { Parallel/Cross-over randomized controlled trials (RCTs) } \\
\text { Controlled clinical trials (CCTs) } \\
\text { Controlled before and after studies } \\
\text { Single-arm interventional studies (within subject control group) } \\
\text { Cohort }\end{array}$ \\
\hline Other & English language \\
\hline
\end{tabular}

\subsection{Selection of Studies}

Two review authors (MK, DJ) independently screened all the titles and abstracts retrieved from the search in order to identify those that may meet the inclusion criteria. They classified studies as being relevant, possibly relevant and irrelevant. Three reviewers (MK, DJ, SKA) independently assessed the full texts of all relevant and possibly relevant studies to assess inclusion. Discrepancies were resolved by referring to a senior review author (ES, SV). 


\subsection{Data Collection}

Standardized data extraction forms were used to extract data from full-text articles. Extracted data included general characteristics of the study (first author, publication year, country, settings, design), sample size, age and sex distribution in groups, diagnosis methods, type of MBI and other relevant data including frequency and duration, control (active or passive), primary outcome, secondary outcomes, primary and secondary measurement tools, length of study, follow up period, statistically significant outcomes, and adverse events reported. Data extraction was completed by one reviewer (DJ) and independently verified by a second reviewer (SKA). Disagreements between the authors were resolved by discussion until consensus was reached; if consensus could not be reached, a senior reviewer's opinion was sought.

\subsection{Data Analysis}

This systematic review was conducted to determine which outcomes and outcome measures were used in the studies of MBIs for the treatment of ME/CFS patients and whether the interventions were effective. General information of the included studies along with the statistically significant and insignificant outcomes were described. We present the findings of studies using different diagnostic criteria (e.g., Oxford criteria, CDC criteria) separately. We also report whether studies assessed adverse events, their absence or presence, and frequencies. A meta-analysis was not performed due to heterogeneous interventions and outcomes used in the included studies. Cochrane risk of bias assessment tool was used by two independent review authors (SKA, SP) to assess sequence generation, allocation concealment, blinding, incomplete outcome data, selective outcome reporting, and other sources of bias [34] in RCTs. Other study designs including single-arm experimental studies were also appraised by two independent reviewers (SKA, SP) for risk of bias using Cochrane Risk of Bias Assessment Tool for Non-Randomized Studies of Intervention (ACROBAT_NRSI) which was recently renamed ROBINS-I [35]. Domains for assessing the risk of bias in these studies include bias due to confounding, selection of participants, measurement of interventions, a departure from the intended intervention, missing data, measurement of outcomes, and selection of the reported result.

\subsection{Patient Involvement}

Patient engagement in health research can improve the quality, relevance and impact of the research $[36,37]$. To recruit patient research partners in this study, a "call for patient representative" letter was developed and distributed among patients, caregivers and advocates. Three patient partners were selected based on their educational background, personal experience, and health status to participate in the study team. They did not receive any financial compensation. They participated regularly in teleconference calls and skype meetings. They also provided feedback and participated in team discussions via email. They contributed to the protocol design, development of the literature search strategy, the condition/diagnosis definitions, and outcome selection.

\section{Results}

Our search results yielded 382 references. After removing duplicates, 270 were screened using title and abstracts, and 47 references were considered relevant for full-text screening. Considering the a priori inclusion criteria and obtaining additional clarifying information from authors of some of the references, twelve studies (17 reports) were ultimately included $[10,28-30,38-45]$. The flow of studies through the screening process of the review is shown in Figure 1. The excluded studies and the reasons for exclusion are shown in Table A2. 


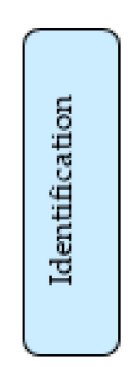

380 references identified through searches

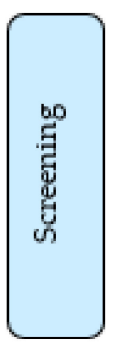

270 were screened
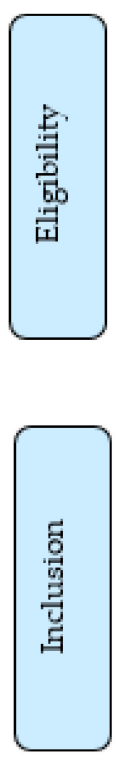

18 references were considered for inclusion, additional information obtained from authors and a total of 12 studies (17 references) were finally included in this systematic review.

223 not relevant

29 excluded for:

- Case study $(\mathrm{n}=1)$

- No population of interest $(\mathrm{n}=12)$

- No eligible control $(\mathrm{n}=6)$

- No intervention of interest $(\mathrm{n}=7)$

- Review ( $\mathrm{n}=1)$

- No peer-reviewed publication $(\mathrm{n}=1)$

- Full-text unavailable $(\mathrm{n}=1)$

Figure 1. Adapted version of PRISMA flow diagram of study selection for the ME/CFS systematic review.

\subsection{Characteristics of the Included Studies}

Table 2 shows the characteristics of all the included studies. 
Table 2. General characteristics of the included studies.

\begin{tabular}{|c|c|c|c|c|c|c|}
\hline First Author, Year, Country & Setting & $\begin{array}{l}\text { Design, Sample Size (En- } \\
\text { rolled/Completed/Analyzed), } \\
\text { Treatment Duration }\end{array}$ & $\begin{array}{l}\text { Study Population (Diagnosis, } \\
\text { Age, Gender) }\end{array}$ & $\begin{array}{c}\text { Mind-Body Intervention, } \\
\text { Frequency, } \\
\text { Duration, } \\
\text { Self-Practice }\end{array}$ & Control Group & Outcome, Measurement Tool and Validity \\
\hline Surawy, Ch., 2005, UK [29] & Not reported & $\begin{array}{c}\text { A series of exploratory studies: } \\
\text { Study } 1 \\
\text { Design: RCT, } \\
\text { Sample size: } \\
\text { Intervention: } 9 / 9 / 9, \\
\text { Control: } 9 / 8 / 8 \\
\text { Treatment duration: } 8 \text { weeks } \\
\text { Study } 2 \\
\text { Design: single-arm trial, } \\
\text { Sample size: } 12 / 9 / 9 \text {,Treatment } \\
\text { duration: } 8 \text { weeks } \\
\text { Study } 3 \\
\text { Design: single-arm trial, } \\
\text { Sample size: } 11 / 9 / 9, \\
\text { Treatment duration: } 8 \text { weeks } \\
\text { and a follow-up period of } 3 \\
\text { months }\end{array}$ & $\begin{array}{c}\text { Patients diagnosed with Oxford } \\
\text { criteria [46] } \\
\text { Study } 1 \\
\text { Age range: } 18-65 \mathrm{y} / \mathrm{o} \\
56 \% \text { female } \\
\text { Study } 2 \\
\text { Age range: } 18-65 \mathrm{y} / \mathrm{o} \\
75 \% \text { female } \\
\text { Study } 3 \\
\text { Age range: } 18-65 \mathrm{y} / \mathrm{o} \\
64 \% \text { female }\end{array}$ & $\begin{array}{c}\text { MBSR/MBCT } \\
\text { Frequency: Once a week, } \\
\text { Duration: Not reported, } \\
\text { Self-practice: Not reported }\end{array}$ & $\begin{array}{l}\text { Study 1: Wait list } \\
\text { Study 2: No control } \\
\text { group } \\
\text { Study 3: No control } \\
\text { group }\end{array}$ & $\begin{array}{c}\text { Study 1, 2, and } 3 \\
\text { Anxiety and Depression: Hospital Anxiety and } \\
\text { Depression Scale (HADS) [47] Fatigue Severity: } \\
\text { Chalder's Fatigue Scale [48] } \\
\text { Quality of Life: SF36 physical functioning [49] } \\
\text { Study } 2 \text { and } 3 \\
\text { Effect of fatigue on quality of life: } \\
\text { Fatigue impact scale [50] }\end{array}$ \\
\hline $\begin{array}{c}\text { Thomas, M., } 2006 \text { and 2008, } \\
\text { UK [39,51] }\end{array}$ & Outpatient clinics & $\begin{array}{c}\text { Design: RCT, } \\
\text { Sample size: } \\
\text { Intervention (relaxation group): } \\
\text { 14/14/14, } \\
\text { Control: } 9 / 9 / 9 \\
\text { Treatment duration: } 10 \text { weeks } \\
\text { Follow-up: } 6 \text { months }\end{array}$ & $\begin{array}{c}\text { Patients diagnosed with CFS by } \\
\text { CDC diagnostic criteria for CFS } \\
\text { [52] } \\
\text { Age (mean } \pm \text { SD): } \\
\text { Intervention (relaxation): } \\
45.7 \pm 12.5 \text {, Control: } \\
46.2 \pm 11.04, \\
\text { Intervention (relaxation): } 71.4 \% \\
\text { female, Control: } 66.7 \% \text { female }\end{array}$ & $\begin{array}{l}\text { Relaxation therapy } \\
\text { Frequency: Once a week, } \\
\text { Duration: } 1 \mathrm{~h}, \\
\text { Self-practice: Not reported }\end{array}$ & Standard medical care & $\begin{array}{c}\text { Report 1 } \\
\text { Illness history: } \\
\text { Beck Depression Inventory [53] } \\
\text { Scale [54], } \\
\text { Chalder Fatigue Scale [48], } \\
\text { Centre for Epidemiological Studion } \\
\text { Cognitive Failures Questionnaire [55], } \\
\text { Current State of Health [5ym, } \\
\text { Fatigue Problem Rating Scale [58], } \\
\text { Cohen-Hob], } \\
\text { Hospital Anxiety and Depression Scale [47], } \\
\text { MOS SF-36 [49] } \\
\text { Perceived Stress [56] } \\
\text { Positive and Negative Affect [59], } \\
\text { Profile of Fatigue Related Symptoms [60], } \\
\text { Sleep Questionnaire [57], } \\
\text { Symptom Check List [57] } \\
\text { Mood testing: Alertness, hedonic tone and anxiety: } \\
\text { measured using 18 computerized visual analogue } \\
\text { mood scales } \\
\text { Performance testing: Word recall, reaction time, } \\
\text { vigilance tasks using a Viglen Dossier laptop } \\
\text { computer connected to a simple 3-button response } \\
\text { box [57] } \\
\text { Report 2 } \\
\text { Primary outcome: Functional performance: } \\
\text { Karnofsky performance scale [61] } \\
\text { Secondary outcome: Global measures of illness and } \\
\text { satisfaction with treatment (including improvement } \\
\text { and changes in fatigue and disability) }\end{array}$ \\
\hline
\end{tabular}


Table 2. Cont

\begin{tabular}{|c|c|c|c|c|c|c|}
\hline First Author, Year, Country & Setting & $\begin{array}{l}\text { Design, Sample Size (En- } \\
\text { rolled/Completed/Analyzed), } \\
\text { Treatment Duration }\end{array}$ & $\begin{array}{l}\text { Study Population (Diagnosis, } \\
\text { Age, Gender) }\end{array}$ & $\begin{array}{l}\text { Mind-Body Intervention, } \\
\text { Frequency, } \\
\text { Duration, } \\
\text { Self-Practice } \\
\end{array}$ & Control Group & Outcome, Measurement Tool and Validity \\
\hline $\begin{array}{l}\text { Bogaerts, K., 2007, } \\
\text { Belgium [38] }\end{array}$ & $\begin{array}{l}\text { University hospital } \\
\text { clinic }\end{array}$ & $\begin{array}{c}\text { Design: Single-arm trial, } \\
\text { Sample size: } 30 / 30 / 30 \\
\text { Treatment duration: Single time } \\
\text { imagery trial }\end{array}$ & $\begin{array}{l}\text { Patients diagnosed with CFS by } \\
\text { CDC diagnostic criteria for } \\
\text { CFS [52] }\end{array}$ & $\begin{array}{l}\text { Relaxation imagery } \\
\text { Frequency: onceDuration: less } \\
\text { than } 5 \text { min Self-practice: NA }\end{array}$ & No control group & $\begin{array}{c}\text { Ventilatory measures: Pet } \mathrm{CO}_{2} \\
\text { Subjective measures: } \\
\text { Degree of fatigue, imagery vividness, concentration } \\
\text { ability on the scripts and similarity of evoked } \\
\text { feelings with daily life feelings: 9-point rating scale } \\
\text { Positive and negative affectivity: Positive and } \\
\text { Negative Affect Schedule (PANAS) [62] } \\
\text { Hyperventilation complaints: Symptom } \\
\text { checklist [63] } \\
\text { Chronic fatigue acceptance: Acceptance Chronic } \\
\text { Fatigue Test (ACFT) } \\
\text { Tendency to worry: Penn-State Worry Questionnaire } \\
\text { (PSWQ) [64] } \\
\text { Valence, arousal and dominance: Self-assessment } \\
\text { Manikin [65] }\end{array}$ \\
\hline Lopez, C., 2011. USA [45] & $\begin{array}{l}\text { Physician referrals, } \\
\text { community }\end{array}$ & $\begin{array}{c}\text { Design: } \mathrm{RCT} \\
\text { Sample size: } 69 / 58 / 58 \\
\text { Treatment duration: } 12 \text { weeks }\end{array}$ & $\begin{array}{c}\text { Patients diagnosed with CFS by } \\
\text { CDC diagnostic criteria for CFS } \\
\text { [52], } \\
\text { Age (mean } \pm \text { SD): } \\
45.9 \pm 9.3 \\
88.4 \% \text { female }\end{array}$ & $\begin{array}{l}\text { Cognitive-behavioral stress } \\
\text { management } \\
\text { Frequency: Weekly } \\
\text { Duration: Two hours } \\
\text { Self-practice: Workbook and } \\
\text { relaxation tapes }\end{array}$ & $\begin{array}{l}\text { Psychoeducation } \\
\text { (half-day seminar) }\end{array}$ & $\begin{array}{l}\text { Distress: Perceived Stress Scale (PSS) [66], Profile of } \\
\text { Mood States (POMS) [67] } \\
\text { Quality of life: Quality of Life Inventory (QOLI) [68] } \\
\text { CFS symptoms: CDC Symptom Inventory for } \\
\text { Chronic Fatigue Syndrome [69] }\end{array}$ \\
\hline
\end{tabular}


Table 2. Cont.

\begin{tabular}{|c|c|c|c|c|c|c|}
\hline First Author, Year, Country & Setting & $\begin{array}{l}\text { Design, Sample Size (En- } \\
\text { rolled/Completed/Analyzed), } \\
\text { Treatment Duration }\end{array}$ & $\begin{array}{l}\text { Study Population (Diagnosis, } \\
\text { Age, Gender) }\end{array}$ & $\begin{array}{c}\text { Mind-Body Intervention, } \\
\text { Frequency, } \\
\text { Duration, } \\
\text { Self-Practice }\end{array}$ & Control Group & Outcome, Measurement Tool and Validity \\
\hline Rimes, K., 2013, U.K. [30] & $\begin{array}{c}\text { A specialist } \\
\text { National Health } \\
\text { Service CFS Unit }\end{array}$ & $\begin{array}{c}\text { Design: RCT, } \\
\text { Sample size: } \\
\text { Intervention group: } 18 / 16 / 16 \\
\text { Control group: } 19 / 19 / 19 \\
\text { Treatment duration: } \\
\text { Introductory session }+8 \text { weeks } \\
\text { Follow-up: at } 2 \text { months, and at } \\
6 \text { months for MBCT group only }\end{array}$ & $\begin{array}{l}\text { Patients diagnosed with CFS by } \\
\text { Fukuda et al. [52] criteria or } \\
\text { Oxford criteria [46] } \\
\text { Age (mean } \pm \text { SD): } \\
\text { Intervention: } 41.4 \pm 10.9 \\
\text { Control: } 45.2 \pm 9.4 \\
\text { Intervention. } 75 \% \text { female, } \\
\text { Control: } 89.5 \% \text { female }\end{array}$ & $\begin{array}{c}\text { MBCT, } \\
\text { Frequency: Once a week, } \\
\text { Duration: } 2.25 \mathrm{~h}, \\
\text { Self-practice: Home practice } \\
\text { with the support of CDs. }\end{array}$ & Waitlist & $\begin{array}{c}\text { Primary outcome: Fatigue: Chalder Fatigue } \\
\text { Scale [48] } \\
\text { Secondary outcomes: } \\
\text { Impairment: The Work and Social Adjustment Scale } \\
\text { [77] Physical Functioning: Physical Functioning } \\
\text { (PF-10) scale) [78,79] } \\
\text { Beliefs about Emotions: Beliefs about Emotions } \\
\text { Scale [80] } \\
\text { Self-Compassion: Self-Compassion Scale [81] } \\
\text { Mindfulness: Five-Facet Mindfulness } \\
\text { Questionnaire [82] } \\
\text { Anxiety and Depression: Hospital Anxiety and } \\
\text { Depression Scale (HADS) [47] } \\
\text { All-or-Nothing Behaviour and Catastrophic } \\
\text { Thinking about Fatigue: five-item subscale of the } \\
\text { Cognitive and Behavior Responses to Symptoms } \\
\text { Questionnaire (Moss-Morris and Chalder, in } \\
\text { preparation; King's College London, UK) } \\
\text { Acceptability and Engagement: } \\
\text { Record of class attendance and amount of home } \\
\text { practice undertaken }\end{array}$ \\
\hline
\end{tabular}


Table 2. Cont.

\begin{tabular}{|c|c|c|c|c|c|c|}
\hline First Author, Year, Country & Setting & $\begin{array}{l}\text { Design, Sample Size (En- } \\
\text { rolled/Completed/Analyzed), } \\
\text { Treatment Duration }\end{array}$ & $\begin{array}{l}\text { Study Population (Diagnosis, } \\
\text { Age, Gender) }\end{array}$ & $\begin{array}{c}\text { Mind-Body Intervention, } \\
\text { Frequency, } \\
\text { Duration, } \\
\text { Self-Practice }\end{array}$ & Control Group & Outcome, Measurement Tool and Validity \\
\hline Oka, T., 2014, Japan [28] & $\begin{array}{l}\text { Outpatients with } \\
\text { CFS who visited the } \\
\text { Department of } \\
\text { Psychosomatic } \\
\text { Medicine of } \\
\text { KyushuUniversity } \\
\text { Hospital }\end{array}$ & $\begin{array}{c}\text { Design: RCT, } \\
\text { Sample size: } \\
\text { Intervention: } 15 / 15 / 15 \\
\text { Control: } 15 / 15 / 15 \\
\text { Treatment duration: } \\
\text { Two months }\end{array}$ & $\begin{array}{c}\text { Patients diagnosed with CFS by } \\
\text { CDC diagnostic criteria for CFS } \\
\text { [52] } \\
\text { Age (mean } \pm \text { SD): } \\
\text { Intervention: } 38.0 \pm 11.1 \\
\text { Control: } 39.1 \pm 14.2 \\
\text { Intervention: } 80 \% \text { female } \\
\text { Control: } 80 \% \text { female }\end{array}$ & $\begin{array}{c}\text { Isometric yoga } \\
\text { Frequency: every two to three } \\
\text { weeks, at least } 4 \text { times during } \\
\text { the intervention period, } \\
\text { Duration: } 20 \text { min, } \\
\text { Self-practice: } \\
\text { With the aid of a digital } \\
\text { videodisk and booklet }\end{array}$ & Waitlist & $\begin{array}{l}\text { Acute effects of isometric yoga on fatigue: The } \\
\text { fatigue and vigor score of the Profile of Mood States } \\
\text { (POMS) questionnaire [67] immediately after the } \\
\text { final 20-min yoga session } \\
\text { Chronic effects of isometric yoga on fatigue: } \\
\text { Chalder's Fatigue Scale [48] } \\
\text { Quality of Life: Medical Outcomes Study Short } \\
\text { Form 8, standard version (SF-8) [87] }\end{array}$ \\
\hline $\begin{array}{c}\text { Oka, T., } 2018 \text { and 2019, Japan } \\
{[42,91]}\end{array}$ & $\begin{array}{l}\text { Outpatients with } \\
\text { CFS who visited the } \\
\text { Department of } \\
\text { Psychosomatic } \\
\text { Medicine of } \\
\text { KyushuUniversity } \\
\text { Hospital }\end{array}$ & $\begin{array}{l}\text { Design: Single-arm trial } \\
\text { Sample size: } 15 \\
\text { Treatment duration: Eight } \\
\text { weeks }\end{array}$ & $\begin{array}{l}\text { Patients diagnosed with CFS by } \\
\text { CDC diagnostic criteria for CFS } \\
\text { [52], the 2011 international } \\
\text { consensus } \\
\text { criteria for myalgic } \\
\text { encephalomyelitis [92] and the } \\
2015 \text { diagnostic criteria for } \\
\text { systemic exertion intolerance } \\
\text { disease [1] } \\
\text { Age (mean } \pm \text { SD): } 38.0 \pm 11.1 \\
80 \% \text { female }\end{array}$ & $\begin{array}{l}\text { Sitting isometric yoga } \\
\text { Frequency: Biweekly with a } \\
\text { yoga instructor } \\
\text { Duration: } 20 \mathrm{~min} \\
\text { Self-practice: Daily in-home } \\
\text { session }\end{array}$ & No control group & $\begin{array}{l}\text { Report 1: } \\
\text { Fatigue and vigor: The fatigue and vigor score of the } \\
\text { Profile of Mood States (POMS) questionnaire [67] } \\
\text { Autonomic nervous system (ANS) functions: Heart } \\
\text { rate and Heart rate variability (HRV) } \\
\text { Blood biomarkers: Serum cortisol, DHEA-S, TNF- } \alpha \text {, } \\
\text { IL-6, IFN- } \alpha \text {, IFN- }-\gamma \text {, PRL, total carnitine, free } \\
\text { carnitine, and acylcarnitine, and plasma TGF- } \beta 1 \text {, } \\
\text { BDNF, MHPG, and HVA } \\
\text { Report 2: } \\
\text { Fatigue severity: Chalder fatigue scale (FS) score [48] } \\
\text { Levels of the blood biomarkers: Cortisol, DHEA-S, } \\
\text { TNF- } \alpha \text {, IL-6, prolactin, carnitine, TGF- } \beta 1 \text {, BDNF, } \\
\text { MHPG, HVA, and } \alpha-\text { MSH } \\
\text { The autonomic nervous functions: Heart rate (HR) } \\
\text { and HR variability } \\
\text { Alexithymia: The 20-item Toronto Alexithymia Scale } \\
\text { (TAS-20) [93] } \\
\text { Anxiety and depression: Japanese version of the } \\
\text { Hospital Anxiety and Depression Scale (HADS) [47] }\end{array}$ \\
\hline
\end{tabular}


Table 2. Cont.

\begin{tabular}{|c|c|c|c|c|c|c|}
\hline First Author, Year, Country & Setting & $\begin{array}{l}\text { Design, Sample Size }(\text { En- } \\
\text { rolled/Completed/Analyzed), } \\
\text { Treatment Duration }\end{array}$ & $\begin{array}{l}\text { Study Population (Diagnosis, } \\
\text { Age, Gender) }\end{array}$ & $\begin{array}{c}\text { Mind-Body Intervention, } \\
\text { Frequency, } \\
\text { Duration, } \\
\text { Self-Practice }\end{array}$ & Control Group & Outcome, Measurement Tool and Validity \\
\hline Jonsjo, M., 2019, Sweden [44] & $\begin{array}{l}\text { Tertiary specialist } \\
\text { clinic }\end{array}$ & $\begin{array}{l}\text { Design: Single-arm trial } \\
\text { Sample size: } 40 / 32 / 32 \\
\text { Treatment duration: } 13 \text { sessions } \\
\text { with three- and six-month } \\
\text { follow-up }\end{array}$ & $\begin{array}{c}\text { Patients diagnosed with CFS } \\
\text { according to CDC [52] and } 2003 \\
\text { Canadian } \\
\text { criteria for ME/CFS }[88] \\
\text { Age (mean } \pm \text { SD): } 49.02 \pm 10.78 \\
76.7 \% \text { female }\end{array}$ & $\begin{array}{l}\text { Acceptance and commitment } \\
\text { therapy } \\
\text { Frequency: Weekly to biweekly } \\
\text { depending on illness severity } \\
\text { (13 sessions) } \\
\text { Duration: } 45 \text { min } \\
\text { Self-practice: Home } \\
\text { assignments }\end{array}$ & No control group & $\begin{array}{c}\text { Primary outcomes: } \\
\text { Disability: The pain disability index [94] } \\
\text { Psychological inflexibility: The Psychological } \\
\text { Inflexibility in Fatigue Scale (PIFS) [95] } \\
\text { Secondary outcomes: } \\
\text { ME/CFS symptoms and severity: 5-point scale } \\
\text { Fatigue: The Multidimensional Fatigue Inventory } \\
\text { (MFI-20) [96] } \\
\text { Anxiety and depression: The Hospital Anxiety and } \\
\text { Depression Scale (HADS) [47] } \\
\text { Dimensions of mental and physical health: SF-36 } \\
\text { Health Survey [79] } \\
\text { Health-related quality of life: EQ-5D-3L [97] }\end{array}$ \\
\hline Takakura, S., 2019, Japan [10] & $\begin{array}{l}\text { Outpatients with } \\
\text { CFS who visited the } \\
\text { Department of } \\
\text { Psychosomatic } \\
\text { Medicine of } \\
\text { Kyushu University } \\
\text { Hospital }\end{array}$ & $\begin{array}{l}\text { Design: Single-arm trial } \\
\text { Sample size: } 9 \\
\text { Treatment duration: Three } \\
\text { months }\end{array}$ & $\begin{array}{l}\text { Patients diagnosed with CFS } \\
\text { according to the 1994 Fukuda } \\
\text { case definition of CFS [52], the } \\
2011 \text { International Consensus } \\
\text { Criteria for ME [92], and the } \\
2015 \text { diagnostic criteria for } \\
\text { systemic exertion intolerance } \\
\text { disease [1] Age (mean } \pm \text { SD): } \\
37.2 \pm 9.9 \\
\text { All female }\end{array}$ & $\begin{array}{l}\text { Recumbent isometric yoga } \\
\text { Frequency: Every two to four } \\
\text { weeks } \\
\text { Duration: } 20-30 \mathrm{~min} \\
\text { depending on patient's } \\
\text { preference } \\
\text { Self-practice: In-home daily } \\
\text { sessions }\end{array}$ & No control group & $\begin{array}{c}\text { Fatigue: Japanese version of } 11 \text { item Chalder Fatigue } \\
\text { Scale }[98,99] \\
\text { Human microRNA }\end{array}$ \\
\hline
\end{tabular}

${ }^{*} 2$ dropped out before the intervention ${ }^{* *} 4$ dropped out before the intervention ${ }^{\wedge} 5$ dropped out before the intervention ${ }^{\wedge} 12$ dropped out before the intervention. 


\subsection{Design}

Six studies were prospective RCTs with at least one eligible control group [28,30,39-41,45]. One manuscript presented a brief report of three studies in which one was a prospective RCT and the other two were single-arm experimental studies [29]. Five additional publications were also single-arm experimental studies [10,38,42-44].

\subsection{Population}

Participants were all adults diagnosed with ME/CFS ( $n=564$ total; sample size range $n=9-150)$. Six studies used the Center for Disease Control and Prevention (CDC) criteria for the diagnosis of their patients [28,39-41,45,51]. One study used the 2003 Canadian criteria [43]. One study used Oxford criteria [29], one study used both CDC and Oxford criteria [30] and three studies used a combination of CDC criteria with 2003 or 2005 versions of Canadian criteria and 2011 international consensus criteria [10,42,44].

The healthcare settings included outpatient settings [28,39], community [40,41,43,45], a university hospital clinic [38], department of psychosomatic medicine [10,42] and a specialist ME/CFS unit $[30,44]$. One study did not report the setting from which their patients were recruited [29].

Three studies were conducted in the United Kingdom [29,30,39], three in Japan [10,28,42], two were conducted in Hong Kong, China [40,41], and one each in Belgium [38], Norway, Sweden, and USA [43-45].

\subsection{Intervention}

A variety of different interventions were implemented in the included studies comprising mindfulness-based stress reduction/mindfulness-based cognitive therapy (MBSR/MBCT) [29], MBCT [30,43], relaxation therapy [39], relaxation imagery [38], Qigong exercise training [41], Baduanjin Qigong [40], and isometric yoga [28], seated isometric yoga [42], recumbent isometric yoga [10], acceptance and commitment therapy [44] and cognitive-behavioral stress management [45]. Treatment duration ranged between 5-12 weeks.

\subsection{Comparison}

Participants assigned to the control group were either placed on the waiting list [28-30,40,41] or received standard medical care [39]. They were advised to keep their usual lifestyle activities including seeking general medical care but not to participate in any activities similar to the intervention of interest.

\subsection{Outcomes}

Many different outcomes and outcome measures were reported in the included studies. Four studies clearly stated their primary and secondary outcomes/objectives [30,39-41]. Fatigue severity was measured by seven studies using Chalder fatigue scale $[10,28-30,40,41,43]$. One study (published as two reports), listed Chalder fatigue scale in one of the reports as the administered questionnaire [51]. In the other report, however, they measured fatigue using patient-rated Likert-type scales [39]. Other studies used either profile of mood state (POMS) [42,45] or multidimensional fatigue inventory (MFI-20) [44].

Eight studies measured anxiety and depression using the Hospital Anxiety and Depression Scale (HADS) $[29,30,40,41,43,44,51,91]$. Six studies measured quality of life or physical and/or mental functioning using different quality of life outcome measures [28-30,41,45,51]. Seven studies measured objective outcomes including ventilatory parameters [38], performance testing by computer programs [51], telomerase activity [70], autonomic nervous system functions, blood biomarkers [42,91], adiponectin levels [83], and microRNA changes [10]. Table 2 describes the details of these outcome measures and the other outcomes measured in the included studies. 


\subsection{Effects of Interventions}

Due to heterogeneous interventions and outcome measures used in the included studies, a meta-analysis was not performed. The statistically significant outcomes reported by these studies are presented in Tables 3 and 4 . Tables A3 and A4 show the statistically insignificant outcomes. 
Table 3. Significant outcomes in the included studies using CDC, Canadian and international consensus criteria for diagnosing CFS.

\begin{tabular}{|c|c|c|c|c|c|c|}
\hline Intervention Type & First Auth & & Outcome (Assessed by) & Comparison Groups & Comparison Time Point & $p$-Value \\
\hline \multirow{10}{*}{ Relaxation-based } & \multirow{9}{*}{$\begin{array}{c}\text { Thomas, M., } 2006 \text { and } 2008 \\
{[39,51]}\end{array}$} & \multirow{3}{*}{ Report 1 (2006) } & $\begin{array}{l}\text { Alertness (as part of a subjective } \\
\text { mood scale) }\end{array}$ & Relaxation group (pre-post) & Post follow-up (6 months) & $<0.027$ \\
\hline & & & $\begin{array}{l}\text { Anxiety (as part of a subjective } \\
\text { mood scale) }\end{array}$ & Relaxation group (pre-post) & Post follow-up (6 months) & $<0.002$ \\
\hline & & & $\begin{array}{l}\text { Current state of health } \\
\text { (self-reporting scale) }\end{array}$ & Relaxation group (pre-post) & Post-treatment (10 weeks) & $\begin{array}{l}\text { Reported significant (value } \\
\text { not reported) }\end{array}$ \\
\hline & & \multirow{6}{*}{ Report 2 (2008) } & $\begin{array}{c}\text { Performance score- } 10 \% \\
\text { improvement (Karnofsky scale) }\end{array}$ & Relaxation group (pre-post) & Post-treatment (10 weeks) & $\begin{array}{c}\text { Reported significant (value } \\
\text { not reported) }\end{array}$ \\
\hline & & & $\begin{array}{l}\text { Global measures of health: } \\
\text { overall condition (Likert-type } \\
\text { scale) }\end{array}$ & Relaxation group (pre-post) & Post-treatment (10 weeks) & $\begin{array}{l}\text { Reported significant (value } \\
\text { not reported) }\end{array}$ \\
\hline & & & $\begin{array}{c}\text { Global measures of health: } \\
\text { Fatigue levels (Likert-type scale) }\end{array}$ & Relaxation group (pre-post) & Post-treatment (10 weeks) & $\begin{array}{c}\text { Reported significant (value } \\
\text { not reported) }\end{array}$ \\
\hline & & & $\begin{array}{l}\text { Global measures of health: } \\
\text { Fatigue levels (Likert-type scale) }\end{array}$ & Relaxation group (pre-post) & Post follow-up (6 months) & $\begin{array}{l}\text { Reported significant (value } \\
\text { not reported) }\end{array}$ \\
\hline & & & $\begin{array}{l}\text { Global measures of health: } \\
\text { reduction in disability } \\
\text { (Likert-type scale) }\end{array}$ & Relaxation group (pre-post) & Post-treatment (10 weeks) & $\begin{array}{l}\text { Reported significant (value } \\
\text { not reported) }\end{array}$ \\
\hline & & & $\begin{array}{l}\text { Global measures of health: } \\
\text { reduction in disability } \\
\text { (Likert-type scale) }\end{array}$ & Relaxation group (pre-post) & Post follow-up (6 months) & $\begin{array}{l}\text { Reported significant (value } \\
\text { not reported) }\end{array}$ \\
\hline & Bogaerts K., 2007 [38] & & $\mathrm{PetCO}_{2}$ & $\begin{array}{l}\text { Relaxation imagery } \\
\text { (pre-post) }\end{array}$ & Post intervention & $<0.01$ \\
\hline \multirow{6}{*}{ Cognitive-based } & \multirow{6}{*}{$\begin{array}{c}\text { Lopez C., } 2011 \text { [45] } \\
\text { (Cognitive restructuring) }\end{array}$} & & Distress (Perceived stress scale) & CBSM compared to control & Time $X$ group ** & 0.03 \\
\hline & & & $\begin{array}{l}\text { Total mood disturbance (Profile } \\
\text { of Mood States (POMS) }\end{array}$ & CBSM compared to control & Time $X$ group ** & 0.05 \\
\hline & & & Quality of life (QOLI Category) & CBSM compared to control & Time $X$ group ** & 0.002 \\
\hline & & & Quality of life (QOLI Raw score) & CBSM compared to control & Time $X$ group ** & 0.05 \\
\hline & & & $\begin{array}{c}\text { Quality of life (QOLI Total } \\
\text { score) }\end{array}$ & CBSM compared to control & Time X group ** & 0.05 \\
\hline & & & $\begin{array}{l}\text { CFS symptoms (Total CDC } \\
\text { symptom severity) }\end{array}$ & CBSM compared to control & Time $X$ group ** & 0.04 \\
\hline
\end{tabular}


Table 3. Cont

\begin{tabular}{|c|c|c|c|c|c|}
\hline Intervention Type & First Author, Year & Outcome (Assessed by) & Comparison Groups & Comparison Time Point & $p$-Value \\
\hline & \multirow{4}{*}{$\begin{array}{l}\text { Sollie K., } 2017 \text { [43] (Mindfulness-based } \\
\text { cognitive therapy) }\end{array}$} & Fatigue (Chalder Fatigue Scale) & MBCT (pre-post) & Post intervention (8 weeks) & $\begin{array}{l}\mathrm{p} \text { value not reported, } \\
\text { medium effect size was } \\
\text { reported }(\mathrm{d}=0.56)\end{array}$ \\
\hline & & Anxiety (HADS) & MBCT (pre-post) & Post intervention (8 weeks) & $\begin{array}{l}\mathrm{p} \text { value not reported, } \\
\text { medium to large effect size } \\
\text { was reported }(\mathrm{d}=0.68)\end{array}$ \\
\hline & & Anxiety (HADS) & MBCT (pre-post) & Post follow-up (3 months) & $\begin{array}{l}\mathrm{p} \text { value not reported, } \\
\text { medium effect size was } \\
\text { reported }(\mathrm{d}=0.48)\end{array}$ \\
\hline & & $\begin{array}{l}\text { Dispositional mindfulness (Five } \\
\text { Facet Mindfulness } \\
\text { questionnaire) }\end{array}$ & MBCT (pre-post) & Post follow-up (3 months) & $\begin{array}{l}\text { p value not reported, large } \\
\text { effect size was reported } \\
(\mathrm{d}=0.77)\end{array}$ \\
\hline & \multirow{10}{*}{ Jonsjo, M., 2019 [44] (Psychological flexibility) } & Disability (Pain disability index) & $\mathrm{ACT}$ (pre-post) & $\begin{array}{l}\text { Post intervention (after } \\
13 \text { sessions) }\end{array}$ & 0.000 \\
\hline & & $\begin{array}{c}\text { Psychological flexibility } \\
\text { (Psychological inflexibility } \\
\text { fatigue scale) }\end{array}$ & ACT (pre-post) & $\begin{array}{l}\text { Post intervention (after } \\
13 \text { sessions) }\end{array}$ & 0.000 \\
\hline & & CFS symptoms & $\mathrm{ACT}$ (pre-post) & $\begin{array}{l}\text { Post intervention (after } \\
13 \text { sessions) }\end{array}$ & 0.017 \\
\hline & & Anxiety (HADS) & $\mathrm{ACT}$ (pre-post) & $\begin{array}{l}\text { Post intervention (after } \\
13 \text { sessions) }\end{array}$ & 0.001 \\
\hline & & General fatigue (MFI-20) & ACT (pre-post) & $\begin{array}{l}\text { Post intervention (after } \\
13 \text { sessions) }\end{array}$ & 0.024 \\
\hline & & General fatigue (MFI-20) & ACT (pre-post) & $\begin{array}{l}\text { Post intervention to post } \\
\text { follow-up ( } 3 \text { months) }\end{array}$ & 0.049 \\
\hline & & Physical fatigue (MFI-20) & ACT (pre-post) & $\begin{array}{l}\text { Post intervention (after } \\
13 \text { sessions) }\end{array}$ & 0.046 \\
\hline & & Mental fatigue (MFI-20) & ACT (pre-post) & $\begin{array}{l}\text { Post intervention (after } \\
13 \text { sessions) }\end{array}$ & 0.004 \\
\hline & & Reduced activity (MFI-20) & ACT (pre-post) & $\begin{array}{l}\text { Post intervention (after } \\
13 \text { sessions) }\end{array}$ & 0.041 \\
\hline & & Reduced motivation (MFI-20) & ACT (pre-post) & $\begin{array}{l}\text { Post intervention (after } \\
13 \text { sessions) }\end{array}$ & 0.043 \\
\hline
\end{tabular}


Table 3. Cont

\begin{tabular}{|c|c|c|c|c|c|c|}
\hline \multirow[t]{2}{*}{ Intervention Type } & \multicolumn{2}{|c|}{ First Author, Year } & \multirow{2}{*}{$\begin{array}{c}\text { Outcome (Assessed by) } \\
\text { SF-36 physical }\end{array}$} & \multirow{2}{*}{$\begin{array}{c}\text { Comparison Groups } \\
\text { ACT (pre-post) }\end{array}$} & \multirow{2}{*}{$\begin{array}{c}\text { Comparison Time Point } \\
\text { Post intervention (after } \\
13 \text { sessions) }\end{array}$} & \multirow{2}{*}{$\begin{array}{c}p \text {-Value } \\
0.009\end{array}$} \\
\hline & & & & & & \\
\hline \multirow{16}{*}{ Movement-based } & \multirow{16}{*}{ Chan J., 2013 [41] } & \multirow{5}{*}{$\begin{array}{c}\text { Ho, R., } 2012 \\
\text { (Preliminary report) }\end{array}$} & $\begin{array}{l}\text { Quality of life: Mental } \\
\text { functioning score (MOS SF-12) }\end{array}$ & Qigong (pre-post) & Post-training (5 weeks) & $<0.01$ \\
\hline & & & $\begin{array}{c}\text { Quality of life: Mental } \\
\text { functioning score (MOS SF-12) }\end{array}$ & Qigong (pre-post) & Post intervention (4 months) & $<0.01$ \\
\hline & & & $\begin{array}{c}\text { Quality of life: Mental } \\
\text { functioning score (MOS SF-12) }\end{array}$ & Qigong compared to control & Time $X$ group $* *$ & 0.001 \\
\hline & & & $\begin{array}{l}\text { Telomerase activity }{ }^{*} \\
\text { (Telomerase PCR ELISA) }\end{array}$ & Qigong (pre-post) & Post intervention (4 months) & $<0.05$ \\
\hline & & & $\begin{array}{l}\text { Telomerase activity }{ }^{*} \\
\text { (Telomerase PCR ELISA) }\end{array}$ & Qigong compared to control & Time $X$ group ** & 0.029 \\
\hline & & \multirow{8}{*}{$\begin{array}{l}\text { Chan J., } 2013 \\
\text { (main report) }\end{array}$} & Total fatigue score (ChFS) & Qigong (pre-post) & Post intervention (4 months) & $<0.001$ \\
\hline & & & Total fatigue score (ChFS) & Qigong compared to control & Time $X$ group ** & 0.000 \\
\hline & & & Physical fatigue score (ChFS) & Qigong (pre-post) & Post intervention (4 months) & $<0.001$ \\
\hline & & & Physical fatigue score (ChFS) & Qigong compared to control & Time $X$ group ** & 0.000 \\
\hline & & & Mental fatigue score (ChFS) & Qigong compared to control & Time $X$ group ** & 0.050 \\
\hline & & & Anxiety score (HADS) & Qigong (pre-post) & Post intervention (4 months) & $<0.001$ \\
\hline & & & Depression score (HADS) & Qigong (pre-post) & Post intervention (4 months) & $<0.001$ \\
\hline & & & Depression score (HADS) & Qigong compared to control & Time $X$ group ** & 0.002 \\
\hline & & \multirow{3}{*}{$\begin{array}{l}\text { Li J., } 2015 \text { (Subset } \\
\text { study report) }\end{array}$} & $\begin{array}{l}\text { Spirituality (the spirituality } \\
\text { subscale of BMSWBI-S) }\end{array}$ & Qigong compared to control & Post intervention (3 months) & 0.013 \\
\hline & & & $\begin{array}{l}\text { Quality of life: mental } \\
\text { component summary (MOS } \\
\text { SF-12) }\end{array}$ & Qigong compared to control & Post intervention (3 months) & 0.002 \\
\hline & & & $\begin{array}{l}\text { Quality of life: mental } \\
\text { component summary (MOS-SF } \\
\text { 12) }\end{array}$ & Qigong compared to control & $\begin{array}{l}\text { Post intervention (change score } \\
\text { from baseline to } 3 \text { months) }\end{array}$ & 0.002 \\
\hline
\end{tabular}


Table 3. Cont.

Outcome (Assessed by)

Comparison Groups

\section{Comparison Time Point}

Post intervention (change score

Baduanjin Qigong from baseline to 9 weeks)

Sleep quality: total score (PSQI) compared to waitlist

$$
\text { Subjective sleep quality (PSQI) }
$$

Baduanjin Qigong

Post intervention (change score

from baseline to 9 weeks)

Subjective sleep quality (PSQI)

Post follow-up (change score compared to waitlis from baseline to 3 months)

Subjective sleep quality (PSQI) $\quad \begin{gathered}\text { Baduanjin Qigong } \\ \text { compared to wait list }\end{gathered}$ Time $X$ group **

Baduanjin Qigong compared to waitlist

Post intervention (change score from baseline to 9 weeks)

\begin{tabular}{cccc}
\hline Sleep latency (PSQI) & $\begin{array}{c}\text { Baduanjin Qigong } \\
\text { compared to waitlist }\end{array}$ & $\begin{array}{c}\text { Post intervention (change score } \\
\text { from baseline to 9 weeks) }\end{array}$ & $<0.05$ \\
\hline Sleep latency (PSQI) & $\begin{array}{c}\text { Baduanjin Qigong } \\
\text { compared to waitlist }\end{array}$ & Time X group ** & 0.044 \\
\hline Sleep duration (PSQI) & $\begin{array}{c}\text { Baduanjin Qigong } \\
\text { compared to waitlist }\end{array}$ & $\begin{array}{c}\text { Post intervention (change score } \\
\text { from baseline to 9 weeks) }\end{array}$ & $<0.05$ \\
\hline Total fatigue score (ChFS) & $\begin{array}{c}\text { Baduanjin Qigong } \\
\text { compared to waitlist }\end{array}$ & $\begin{array}{c}\text { Post intervention (change score } \\
\text { from baseline to 9 weeks) }\end{array}$ & $<0.001$ \\
\hline Total fatigue score (ChFS) & $\begin{array}{c}\text { Baduanjin Qigong } \\
\text { compared to waitlist }\end{array}$ & $\begin{array}{c}\text { Post follow-up (change score } \\
\text { from baseline to 3 months) }\end{array}$ & $<0.001$ \\
\hline Total fatigue score (ChFS) & $\begin{array}{c}\text { Baduanjin Qigong } \\
\text { compared to waitlist }\end{array}$ & Time X group ** & $<0.001$ \\
\hline Physical fatigue score (ChFS) & $\begin{array}{c}\text { Baduanjin Qigong } \\
\text { compared to waitlist }\end{array}$ & $\begin{array}{c}\text { Post intervention (change score } \\
\text { from baseline to 9 weeks) }\end{array}$ & $<0.001$ \\
\hline Physical fatigue score (ChFS) & $\begin{array}{c}\text { Baduanjin Qigong } \\
\text { compared to waitlist }\end{array}$ & $\begin{array}{c}\text { Post follow-up (change score } \\
\text { from baseline to 3 months) }\end{array}$ & $<0.001$ \\
\hline
\end{tabular}

Chan, J. 2014 [40] and Chan, J. 2017 [83]
Report 1 (2014)

\section{$p$-Value}

$<0.05$

$<0.01$

$<0.01$

002


Table 3. Cont

Outcome (Assessed by)

Physical fatigue score (ChFS)

Mental fatigue score (ChFS)

Mental fatigue score (ChFS)

Mental fatigue score (ChFS)

Anxiety (HADS)

Anxiety (HADS)

Anxiety (HADS)

Depression (HADS)

ncrease in adiponectin levels

Report 2 (2017)

\begin{tabular}{cc} 
Depression (HADS) & $\begin{array}{c}\text { Baduanjin Qigong } \\
\text { compared to waitlist }\end{array}$ \\
\hline Anxiety (HADS) & Baduanjin Qigong
\end{tabular}

Comparison Groups

Baduanjin Qigong compared to waitlist Baduanjin Qigong

\section{Baduanjin Qigong} compared to waitlis

Baduanjin Qigong compared to waitlist

Baduanjin Qigong compared to waitlist Baduanjin Qigong Baduanjin Qigong compared to waitlist Baduanjin Qigong compared to waitlist Baduanjin Qigong compared to waitlist Baduanjin Qigong compared to waitlist Baduanjin Qigong compared to waitlist

\section{Comparison Time Point}

Time $X$ group **

Post intervention (change score

from baseline to 9 weeks)

Post follow-up (change score

from baseline to 3 months)

Time X group **

Post intervention (change score from baseline to 9 weeks)

Post follow-up (change score

from baseline to 3 months)

Time $X$ group **

Post intervention (change score from baseline to 9 weeks)

$p$-Value

$<0.001$

$<0.001$

$<0.01$

$<0.001$

$<0.01$

$<0.05$

0.016

$<0.001$

$$
\text { Time X group ** }
$$

Post intervention (change score

$$
\text { from baseline to } 9 \text { weeks) }
$$

Post intervention (change score from baseline to 9 weeks)

Post intervention (9 weeks)

core

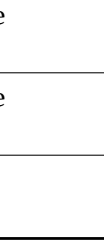

$<0.001$

$<0.05$ 
Table 3. Cont

\begin{tabular}{|c|c|c|c|c|c|c|}
\hline \multirow[t]{11}{*}{ Intervention Type } & \multicolumn{2}{|c|}{ First Author, Year } & \multirow{2}{*}{$\begin{array}{c}\text { Outcome (Assessed by) } \\
\text { Fatigue score -acute effect } \\
\text { (POMS) }\end{array}$} & \multirow{2}{*}{$\begin{array}{c}\text { Comparison Groups } \\
\text { Isometric yoga (pre-post) }\end{array}$} & \multirow{2}{*}{$\begin{array}{c}\text { Comparison Time Point } \\
\begin{array}{c}\text { Before to after the final 20-min } \\
\text { session }\end{array}\end{array}$} & \multirow{2}{*}{$\begin{array}{l}p \text {-Value } \\
<0.001\end{array}$} \\
\hline & \multirow{10}{*}{\multicolumn{2}{|c|}{ Oka T, 2014 [28] }} & & & & \\
\hline & & & Vigor score- acute effect (POMS) & Isometric yoga (pre-post) & $\begin{array}{l}\text { Before to after the final 20-min } \\
\text { session }\end{array}$ & $<0.01$ \\
\hline & & & Physical fatigue score (ChFS) & Isometric yoga (pre-post) & Post intervention (2 months) & 0.004 \\
\hline & & & Physical fatigue score (ChFS) & $\begin{array}{l}\text { Isometric yoga compared to } \\
\text { control }\end{array}$ & Time $X$ group $* *$ & 0.009 \\
\hline & & & Mental fatigue score (ChFS) & Isometric yoga (pre-post) & Post intervention (2 months) & 0.004 \\
\hline & & & Mental fatigue score (ChFS) & $\begin{array}{l}\text { Isometric yoga compared to } \\
\text { control }\end{array}$ & Time $X$ group $* *$ & 0.007 \\
\hline & & & Total fatigue score (ChFS) & Isometric yoga (pre-post) & Post intervention (2 months) & 0.002 \\
\hline & & & Total fatigue score (ChFS) & $\begin{array}{l}\text { Isometric yoga compared to } \\
\text { control }\end{array}$ & Time $X$ group ** & 0.003 \\
\hline & & & $\begin{array}{l}\text { Quality of life: bodily pain } \\
\text { (SF-8) }\end{array}$ & Isometric yoga (pre-post) & Post intervention (2 months) & 0.0001 \\
\hline & & & $\begin{array}{l}\text { Quality of life: general health } \\
\text { perception (SF-8) }\end{array}$ & Isometric yoga (pre-post) & Post intervention ( 2 months) & 0.0021 \\
\hline & \multirow{5}{*}{\multicolumn{2}{|c|}{$\begin{array}{c}\text { Oka, T., } 2018 \text { and Oka, T., } \\
2019[42,91]\end{array}$}} & Fatigue (POMS) & $\begin{array}{l}\text { Acute effects of sitting } \\
\text { isometric yoga (pre-post) }\end{array}$ & $\begin{array}{l}\text { Before to after the final 20-min } \\
\text { session }\end{array}$ & 0.001 \\
\hline & & & Vigor (POMS) & $\begin{array}{c}\text { Acute effects of sitting } \\
\text { isometric yoga (pre-post) }\end{array}$ & $\begin{array}{l}\text { Before to after the final 20-min } \\
\text { session }\end{array}$ & 0.002 \\
\hline & & & Decreased heart rate & $\begin{array}{l}\text { Acute effects of sitting } \\
\text { isometric yoga (pre-post) }\end{array}$ & $\begin{array}{l}\text { Before to after the final 20-min } \\
\text { session }\end{array}$ & 0.047 \\
\hline & & & $\begin{array}{c}\text { Increased high-frequency power } \\
\text { of HR variability }\end{array}$ & $\begin{array}{l}\text { Acute effects of sitting } \\
\text { isometric yoga (pre-post) }\end{array}$ & $\begin{array}{l}\text { Before to after the final 20-min } \\
\text { session }\end{array}$ & 0.028 \\
\hline & & & $\begin{array}{c}\text { Increased serum levels of } \\
\text { DHEA-S }\end{array}$ & $\begin{array}{l}\text { Acute effects of sitting } \\
\text { isometric yoga (pre-post) }\end{array}$ & $\begin{array}{l}\text { Before to after the final 20-min } \\
\text { session }\end{array}$ & 0.012 \\
\hline
\end{tabular}


Table 3. Cont

Outcome (Assessed by)

Comparison Groups

Comparison Time Point

Acute effects of sitting Before to after the final 20-min

session

isometric yoga (pre-post)

ter the final 20-min

Acute effects of sitting

session

Longitudinal effects of

$$
\text { session }
$$

Post intervention (2 months)

Report 2 (2019)

Fatigue (POMS)

$$
\text { (pre-post) }
$$

Longitudinal effects of

sitting isometric yoga

Post intervention (2 months)

0.02

Depression (HADS)

$$
\text { (pre-post) }
$$

Fatigue (11 score Chalder's Recumbent isometric yoga

fatigue scale)

(pre-post)

Post intervention (3 months)

$<0.0001$

Takakura, S., 2019 [10] Changes in miRNA expression $\begin{array}{r}\text { Recumbent isomet } \\ \text { (pre-post) }\end{array}$

Post intervention (3 months)

$<0.05$ (Four miRNAs

significantly upregulated

and 42 were significantly downregulated)

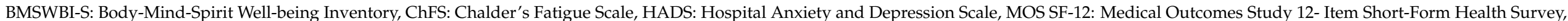

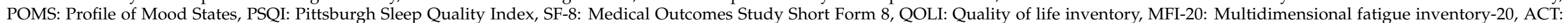

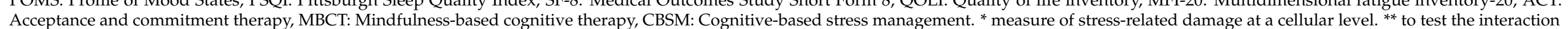
Acceptance and commitmen
effect of time and group. 
Table 4. Significant outcomes in the included studies using Oxford criteria for diagnosing CFS.

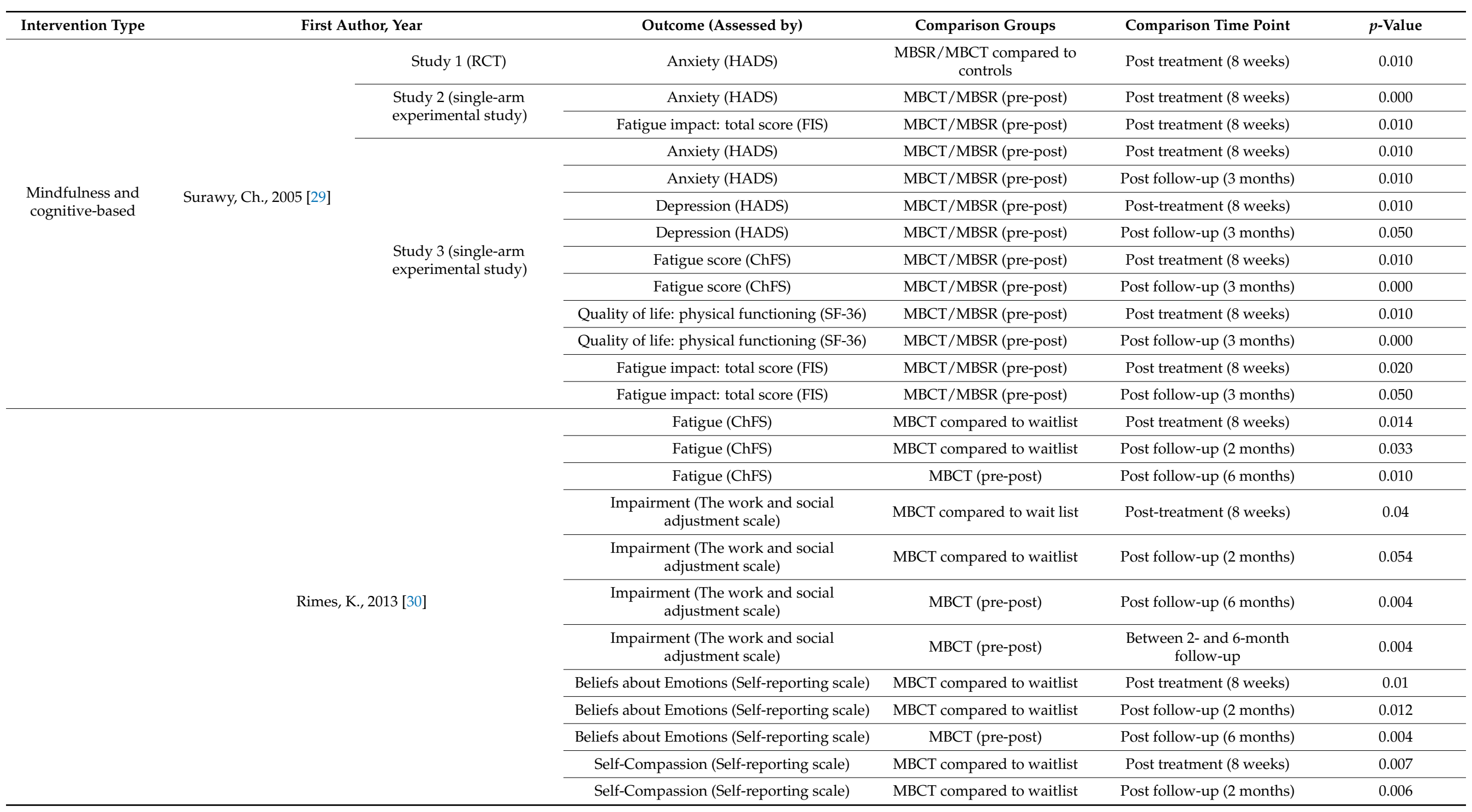


Table 4. Cont.

\begin{tabular}{|c|c|c|c|c|c|}
\hline Intervention Type & First Author, Year & Outcome (Assessed by) & Comparison Groups & Comparison Time Point & $p$-Value \\
\hline & & Self-Compassion (Self-reporting scale) & MBCT (pre-post) & Post follow-up (6 months) & 0.003 \\
\hline & & $\begin{array}{l}\text { Mindfulness ( } 5 \text { facet mindfulness } \\
\text { questionnaire) }\end{array}$ & MBCT compared to waitlist & Post follow-up (2 months) & 0.035 \\
\hline & & $\begin{array}{l}\text { Mindfulness ( } 5 \text { facet mindfulness } \\
\text { questionnaire) }\end{array}$ & MBCT (pre-post) & $\begin{array}{l}\text { Between 2- and 6-month } \\
\text { follow-up }\end{array}$ & 0.017 \\
\hline & & Catastrophizing (Self-reporting scale) & MBCT compared to waitlist & Post treatment (8 weeks) & 0.004 \\
\hline & & Catastrophizing (Self-reporting scale) & MBCT (pre-post) & Post follow-up (6 months) & 0.012 \\
\hline & & $\begin{array}{l}\text { All-or-nothing behavior (Self-reporting } \\
\text { scale) }\end{array}$ & MBCT compared to waitlist & Post treatment (8 weeks) & 0.005 \\
\hline & & $\begin{array}{l}\text { All-or-nothing behavior (Self-reporting } \\
\text { scale) }\end{array}$ & MBCT (pre-post) & Post follow-up (6 months) & 0.017 \\
\hline & & Depression (HADS) & MBCT compared to waitlist & Post-treatment (8 weeks) & 0.038 \\
\hline
\end{tabular}

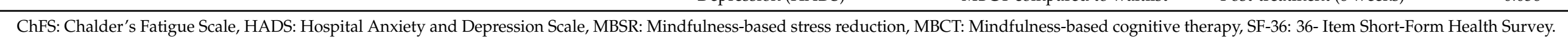


In comparison to the control group, both mental and physical fatigue scores improved significantly in four included studies using MBCT [30], isometric yoga [28], Qigong exercise [41] and Baduanjin Qigong [40]. Two studies showed within-group fatigue improvement in participants receiving an 8-week mindfulness therapy [29] and in participants receiving a 10-week relaxation program [39] (Tables 3 and 4)

Anxiety and depression were improved in participants receiving Baduanjin Qigong compared to the controls after 16 sessions (9 weeks) of therapy [40]. Depression was improved in participants after 4 months of Qigong exercise [41] and 8 weeks of MBCT [30] compared to the control groups. Surawy et al. [29] also showed improvement of anxiety after 8 weeks of MBSR/MBCT intervention compared to the control group.

In comparison to the control group, quality of life improved in participants receiving Qigong exercise [41,70,71] and cognitive-behavioral stress management [45].

Tables 3 and 4 show the details of all the significant outcomes of the included studies according to the diagnosis of ME/CFS (Oxford or CDC criteria).

\subsection{Adverse Events}

Seven studies assessed adverse events: Four did not identify any adverse events [30,39,41,43]; and three studies recorded adverse events such as deterioration of their symptoms, muscle ache, palpitation, dizziness, knee pain, backache, fatigue, and nervousness [28,40,44]. Five studies did not report if they assessed adverse events $[10,29,38,42,45]$

\subsection{Risk of Bias in the Included Studies}

All the included RCT studies were assessed at a high risk of bias in relation to the lack of blinding of participants and personnel. We were not able to assess the risk of bias in many areas as most of the studies were poorly reported (Figure 2). The risk of bias assessment for the single-arm experimental studies using the ROBINS-I assessment tool is shown in Table A5.

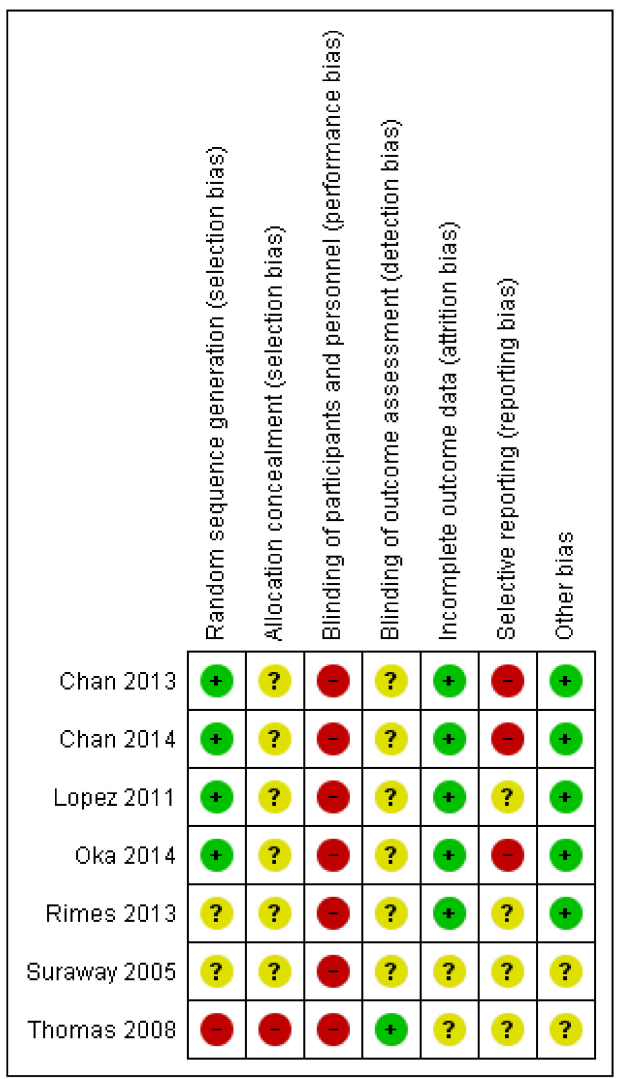

Figure 2. Risk of bias summary: review authors' judgments about each risk of bias item for each included study. 


\section{Discussion}

This is the first systematic review of studies using MBIs in patients with ME/CFS. The MBIs used in these studies were mindfulness-based stress reduction and mindfulness-based cognitive therapy, relaxation, Qigong, and yoga.

The etiology and pathogenesis of ME/CFS are still unknown [1]. Researchers have shown changes in some biological markers [100-103]. Other studies highlight changes in the hypothalamic-pituitary-adrenal (HPA) axis in these patients [104].

It was also suggested that ME/CFS may be a neurophysiological disorder in the brain caused by repeated incidental or unnecessary stimuli in the limbic system, which is known as the threat response/protection center. These stimuli can be emotional, psychological, chemical, and/or physiological and they can keep the threat response center on a continuous high alert [105]. Connections between the amygdala and sympathetic, hypothalamic and other limbic brain systems can initiate a series of stimulations and uncontrolled reactions throughout the whole body, which could be considered as the root cause of CFS symptoms [105].

With increasing knowledge based on neuroplasticity and the impact of limbic function on somatic symptoms, the potential mechanisms of MBIs might be explained. There is growing interest in using MBIs and many programs are being offered directly to the public to assist with mental and physical health. One of these programs developed specifically for ME/CFS (25) has shown modest success in functional ability in a clinical audit. Because patients are accessing MBI programs, there is an urgent need for evidence as to whether these programs are having an impact on the core symptoms of ME/CFS or mainly address the secondary dissatisfaction that comes with having a chronic, poorly understood disease for which there is no cure. In this review, the MBIs used in the included studies were quite heterogenous. Two studies used relaxation techniques, five studies used movement-based therapies including different forms of yoga and Qigong and the remaining ones used various forms of mindfulness and cognitive-based approaches. Table A6 describes these interventions briefly.

In this systematic review, we found the most commonly measured outcomes were fatigue severity, anxiety and depression, and quality of life or its components (e.g., physical and mental functioning). When compared to the control group, fatigue severity, mental functioning and anxiety/depression mostly improved in patients receiving MBIs. However, poor reporting, small sample sizes, different diagnostic criteria, and a high risk of bias may challenge this result. It is also worth noting that these symptoms are not specific and can be found not only in some individuals with ME/CFS but also in individuals with many other physical and mental health conditions.

According to the 2015 Institute of Medicine report [1], impaired function, postexertional malaise and unrefreshing sleep are the core symptoms in ME/CFS patients. None of our included studies, however, measured post-exertional malaise. One study measured sleep using a self-reporting scale which improved after 9 weeks of Qigong exercise [40]. Physical or mental functioning and functional performance were mostly measured using self-report scales and only one study measured performance using objective measures [51].

In contrast, anxiety and depression and some cognitive constructs were commonly measured in the included studies. While these symptoms are important, they are secondary and not the key features of ME/CFS. Reporting secondary outcomes while omitting measurement of the core symptoms of a disease may lead to inaccurate conclusions about treatment effectiveness.

Previous studies have used a variety of definitions for the diagnosis of ME/CFS. Lack of consensus and competing definitions act as a barrier for research in this field. Most of the studies in this systematic review used the 1994 CDC criteria for the diagnosis of ME/CFS and two studies used Oxford criteria.

The Oxford criteria were developed at a consensus meeting [46]. They do not require the presence of any symptom other than disabling fatigue. The presence of other symp- 
toms such as immune, autonomic and mood symptoms differentiate ME/CFS from other common medical and psychiatric conditions including major depression. It has long been suspected that the Oxford criteria may therefore fail to exclude individuals with other fatiguing conditions $[14,19]$.

To address this concern, the Agency for Healthy Research and Quality (AHRQ) in the United States conducted a sensitivity analysis in which the outcomes of treatment studies using the Oxford criteria were compared with studies using other criteria (mostly the 1994 CDC Criteria) [14]. They found that whereas most studies using the Oxford criteria showed some benefits for CBT, studies using the CDC criteria were mixed with no overall benefit. With regards to graded exercise therapy, exclusion of the trials using the Oxford case definition left insufficient evidence about the effectiveness of graded exercise therapy on any outcome. Studies of other therapies were not affected as primary studies had small sample sizes and a high risk of bias. These findings confirm that the choice of inclusion criteria impacts study outcomes. The AHRQ concluded that future research should retire the use of the Oxford case definition. The National Institutes of Health held a consensus workshop to guide the future of ME/CFS research [19]. For similar reasons as the AHRQ, they also recommended that the Oxford Criteria should be retired.

The 1994 CDC criteria also have significant drawbacks. They require four out of eight criteria but none are mandatory. This means two subjects identified with these criteria may have no symptoms in common with each other-one might have four and the other, another four. Moreover, minor symptoms may overlap with the symptoms of psychiatric disorders including major depression [14].

The Institute of Medicine [1] has proposed diagnostic criteria which are very similar to the Canadian Consensus Criteria [88]. They require patients to have moderate, substantial or severe disabling fatigue, post-exertional malaise and unrefreshing sleep for at least half of the time and one of the cognitive impairments or orthostatic intolerance symptoms. Conclusions about the effectiveness of interventions will be possible once studies use the same diagnostic criteria and measure core outcomes using standardized measures.

\subsection{Strengths and Limitations}

Assessment of a broad range of mind-body approaches and outcomes in a systematic fashion was one of the main strengths of this systematic review. Engaging patients in the process of designing the review protocol and in reviewing the findings increase the applicability and relevance of the findings of this study.

As we found a diverse range of interventions and outcomes across the included studies; we were not able to perform a meta-analysis. We also may have missed some relevant information by including only studies published in the English language.

\subsection{Research Implications}

1. As recommended by the Institute of Medicine report, using objective measures is a priority in studies of ME/CFS. There are several symptoms such as post-exertional malaise, cognitive dysfunction, orthostatic intolerance, and changes including impaired immune function and abnormal brain functions that could be measured objectively.

2. Future RCTs will benefit from larger sample sizes. Investigators must use an appropriate randomization method and ensure outcome assessors are blinded to the group identity of the participants. They should measure and report the outcomes specified in their protocol in order to avoid selective reporting.

\section{Conclusions}

In this systematic review, we described the current literature on MBIs for the treatment of ME/CFS. Future clinical trials will benefit from the findings of this study in terms of what outcomes and outcome measures are mostly used in previous studies. We showed that the included studies did not report measuring post-exertional malaise as a core outcome of ME/CFS. On the other hand, fatigue severity, anxiety/depression and mental functioning 
were shown to be improved in the patients receiving MBIs. However, poor reporting, small sample sizes, different diagnostic criteria, and a high risk of bias may challenge this result. We highlight the need for further research to use objective and standardized outcomes and outcome measures for making definitive conclusions.

Author Contributions: Conceptualization, S.V., E.S., and K.O; methodology, S.K.A., M.K., E.S., S.P., D.R.J., T.K., M.P., L.S., K.O. and S.V.; validation, S.K.A., M.K., E.S., D.R.J., S.P. and S.V.; formal analysis, S.K.A.; investigation, S.K.A., M.K., D.R.J. and E.S.; resources, S.V.; data curation, S.K.A.; writing—original draft preparation, S.K.A., M.K. and E.S.; writing—review and editing, S.P., E.S. and S.V.; visualization, S.K.A.; supervision, S.V.; project administration, S.P.; funding acquisition, S.V. All authors have read and agreed to the published version of the manuscript.

Funding: This research was funded by the University of Calgary.

Acknowledgments: The authors thank Susanne King-Jones for her help in the search strategy and contributions to the project.

Conflicts of Interest: The authors declare no conflict of interest. The funders had no role in the design of the study; in the collection, analyses, or interpretation of data; in the writing of the manuscript, or in the decision to publish the results.

\section{Appendix A}

Table A1. Case definitions for the diagnosis of ME/CFS over time.

\begin{tabular}{|c|c|c|}
\hline Advisor Group, Year & Identifier & Case Definition and Required Symptom(s) \\
\hline \multicolumn{3}{|l|}{ For Adults } \\
\hline $\begin{array}{l}\text { Holmes et al., } 1988 \\
\text { (CDC) [17] }\end{array}$ & CFS & $\begin{array}{l}\text { Major criteria } \\
\text { New onset of persistent or relapsing, debilitating fatigue or easy fatigability in a person who has no } \\
\text { previous history of similar symptoms, that does not resolve with bedrest, and that is severe enough to } \\
\text { reduce or impair average daily activity below } 50 \% \text { of the patient's premorbid activity level for a period of } \\
\text { at least } 6 \text { months } \\
\text { Minor criteria } \\
\text { Mild fever } \\
\text { Sore throat } \\
\text { Painful lymph node in the anterior or posterior cervical or axillary distribution } \\
\text { Unexplained generalized muscle weakness } \\
\text { Muscle discomfort or myalgia } \\
\text { Prolonged generalized fatigue ( } \geq 24 \mathrm{~h} \text { ) after normal level of exercise } \\
\text { Migratory arthralgia without joint swelling or redness } \\
\text { Neurological complains one or more of: photophobia, transient visual scotomata, forgetfulness, excessive } \\
\text { irritability, confusion, difficulty thinking, inability to concentrate, depression } \\
\text { Sleep disturbances }\end{array}$ \\
\hline $\begin{array}{l}\text { Sharp et al., } 1991 \\
\text { (Oxford) [46] }\end{array}$ & CFS & $\begin{array}{l}\text { Fatigue as the principal symptom } \\
\text { A definite onset that is not lifelong } \\
\text { The fatigue is severe, disabling, and affects physical and mental functioning } \\
\text { The fatigue should have been present for a minimum of } 6 \text { months during which it was present for more } \\
\text { than } 50 \% \text { of the time } \\
\text { Other symptoms may be present, particularly myalgia, mood and sleep disturbance. }\end{array}$ \\
\hline $\begin{array}{l}\text { Fukuda et al., 1994, } \\
\text { (CDC) [52] }\end{array}$ & CFS & $\begin{array}{l}\text { Clinically evaluated, "unexplained", persistent or relapsing fatigue for } \geq 6 \text { months. } \\
\text { Not the result of ongoing exertion } \\
\text { Not substantially alleviated by rest } \\
\text { Resulting in a substantial reduction in previous activity level. } \\
\text { Four or more of the following concurrently present for } \geq 6 \text { months: } \\
\text { impaired memory or concentration } \\
\text { sore throat } \\
\text { tender cervical or axillary lymph nodes } \\
\text { muscle pain } \\
\text { multi-joint pain } \\
\text { new headaches } \\
\text { unrefreshing sleep } \\
\text { post-exertion malaise }\end{array}$ \\
\hline
\end{tabular}


Table A1. Cont.

\begin{tabular}{lll}
\hline Advisor Group, Year & Identifier & $\begin{array}{l}\text { Case Definition and Required Symptom(s) } \\
\text { These three criteria must all be present for a diagnosis of M.E./PVFS } \\
\text { Exercise-induced fatigue precipitated by trivially small exertion -physical or mental -relative to the } \\
\text { patient's previous exercise tolerance }\end{array}$ \\
London criteria-V2, & Impairment of short-term memory and loss of powers of concentration, usually coupled with other \\
(Dowsett et al., 1994) & neurological and psychological disturbances such as emotional lability, nominal dysphasia, disturbed \\
[106] & sleep patterns, disequilibrium or tinnitus \\
& Fluctuation of symptoms, usually precipitated by either physical or mental exercise
\end{tabular}

For a diagnosis of CFS/ME, a patient must meet the following criteria 1-6 and adhere to item 7: Fatigue

Post-exertional malaise and/or fatigue

Sleep dysfunction

Pain

Canadian Consensus

Criteria,

(Carruthers et al., 2003)

[88]
Two or more of the following neurological/cognitive manifestations:

Confusion

Impairment of concentration and short-term memory consolidation

ME/PVFS Disorientation

difficulty with information processing

categorizing and word retrieval

perceptual and sensory disturbances

One or more symptoms from two of the following categories:

Autonomic manifestation (e.g., orthostatic intolerance, postural orthostatic tachycardia syndrome, ... )

Neuroendocrine manifestation (e.g., loss of thermostatic stability, sweating episode, ... )

Immune manifestation (e.g., tender lymph nodes, recurrent sore throat, ... )

Illness lasting $\geq 6$ months

Definition of Research CFS/ME criteria:

Over the past 6 months, persistent or recurring chronic fatigue that is not lifelong and results in substantial reductions in previous levels of occupational, educational, social and personal activities Post-exertional malaise and/or fatigue

Unrefreshing sleep or disturbance of sleep quantity or rhythm disturbance

Pain (or discomfort) that is often widespread and migratory in nature. At least one symptom from any of the following:

Myofascial and/or joint pain (e.g., deep pain, abdomen/stomach pain, or achy and sore muscles.

Pain, stiffness, or tenderness may occur in any joint but must be present in more than one joint and lacking edema or other signs of inflammation)

Abdominal and/or head pain (e.g., stomach pain or chest pain).

Headaches often described as localized behind the eyes or in the back of the head (includes headaches localized elsewhere, including migraines; headaches would need to be more frequent than they were before, which would indicate a new pattern of a new type as compared to headaches previously experienced (i.e., location of pain has changed, nature of pain has changed), or different in severity type as compared to headaches previously experienced by the patient)

Two or more of the following neurological/cognitive manifestations:

Impaired memory (self-reported or observable disturbance in the ability to recall information or events on a short-term basis)

Difficulty focusing vision and attention (disturbed concentration may impair the ability to remain on task, to screen out extraneous/excessive stimuli)

Revised Canadian

Consensus

Criteria, (Jason et al., 2010) [107]

\section{$\mathrm{ME} / \mathrm{CFS}$}

Difficulty finding the right word

Frequently forget what wanted to say

Absent-mindedness

Slowness of thought

Difficulty recalling information

Need to focus on one thing at a time

Trouble expressing thought

Difficulty comprehending information

Frequently lose train of thought

Sensitivity to bright lights or noise

Muscle weakness/muscle twitches

At least one symptoms from two of the following categories:

Autonomic manifestation: Neurally mediated hypotension, postural orthostatic tachycardia, delayed postural hypotension, palpitations with or without cardiac arrhythmias, dizziness or fainting, feeling unsteady on the feet-disturbed balance, shortness of breath, nausea, bladder dysfunction, or irritable bowel syndrome

Neuroendocrine manifestation: Recurrent feelings of feverishness and cold extremities, subnormal body temperature and marked diurnal fluctuations, sweating episodes, intolerance of extremes of heat and cold, marked weight change-loss of appetite or abnormal appetite

Immune manifestation: Recurrent flu-like symptoms, non-exudative sore or scratchy throat, repeated fevers and sweats, lymph nodes tender to palpitation-generally minimal swelling observed, new sensitivities to food, odors, or chemicals 
Table A1. Cont.

Advisor Group, Year $\quad$ Identifier $\quad$ Case Definition and Required Symptom(s)

Myalgic encephalomyelitis is an acquired neurological disease with complex global dysfunctions. Pathological dysregulation of the nervous, immune and endocrine systems, with impaired cellular energy metabolism and ion transport, are prominent features. Although signs and symptoms are dynamically interactive and causally connected, the criteria are grouped by regions of pathophysiology to provide general focus. A patient will meet the following criteria

A. Post-exertional neuro-immune exhaustion (PENEpen'-e): Compulsory

This cardinal feature is a pathological inability to produce sufficient energy on demand with prominent symptoms primarily in the neuro-immune regions. Characteristics are as follows:

1. Marked, rapid physical and/or cognitive fatigability in response to exertion, which may be minimal such as activities of daily living or simple mental tasks, can be debilitating and cause a relapse

2. Post-exertional symptom exacerbation: e.g., acute flu-like symptoms, pain and worsening of other symptoms.

3. Post-exertional exhaustion may occur immediately after activity or be delayed by hours or days.

4. Recovery period is prolonged, usually taking 24-h or longer. A relapse can last days, weeks or longer.

5. Low threshold of physical and mental fatigability (lack of stamina) results in a substantial reduction in pre-illness activity level.

B. Neurological impairments: At least one symptom from three of the following four symptom categories

1. Neuro-cognitive impairments

a. Difficulty processing information: slowed thought, impaired concentration, e.g., confusion disorientation, cognitive overload, difficulty with making decisions, slowed speech, acquired or exertional dyslexia

b. Short-term memory loss: e.g., difficulty remembering what one wanted to say, what one was saying, retrieving words, recalling information, poor working memory

2. Pain

a. Headaches: e.g., chronic, generalized headaches often involve aching of the eyes, behind the eyes or back of the head that may be associated with cervical muscle tension; migraine; tension headaches

International

Consensus

Criteria,

(Carruthers et al., 2011)

b. Significant pain can be experienced in muscles, muscle-tendon junctions, joints, abdomen or chest. It is non-inflammatory in nature and often migrates, e.g., generalized hyperalgesia, widespread pain (may meet fibromyalgia criteria), myofascial or radiating pain

a. Disturbed sleep patterns: e.g., insomnia, prolonged sleep including naps, sleeping most of the day and being awake most of the night, frequent awakenings, awaking much earlier than before illness onset, vivid dreams/nightmares

b. Unrefreshed sleep: e.g., awaken feeling exhausted regardless of the duration of sleep, day-time sleepiness

4. Neuro-sensory, perceptual and motor disturbances

a. Neurosensory and perceptual: e.g., inability to focus vision, sensitivity to light, noise, vibration, odor, taste and touch; impaired depth perception

b. Motor: e.g., muscle weakness, twitching, poor coordination, feeling unsteady on feet, ataxia

c. Immune, gastro-intestinal and genitourinary Impairments: At least one symptom from three of the following five symptom categories

1. Flu-like symptoms may be recurrent or chronic and typically activate or worsen with exertion. e.g., sore throat, sinusitis, cervical and/or axillary lymph nodes may enlarge or be tender on palpitation

2. Susceptibility to viral infections with prolonged recovery periods

3. Gastro-intestinal tract: e.g., nausea, abdominal pain, bloating, irritable bowel syndrome

4. Genitourinary: e.g., urinary urgency or frequency, nocturia

5. Sensitivities to food, medications, odors or chemicals

d. Energy production/transportation impairments: At least one symptom

1. Cardiovascular: e.g., inability to tolerate an upright position-orthostatic intolerance, neurally mediated hypotension, postural orthostatic tachycardia syndrome, palpitations with or without cardiac arrhythmias, light-headedness/dizziness

2. Respiratory: e.g., air hunger, labored breathing, fatigue of chest wall muscles

3. Loss of thermostatic stability: e.g., subnormal body temperature, marked diurnal fluctuations; sweating episodes, recurrent feelings of feverishness with or without low-grade fever, cold extremities

4. Intolerance of extremes of temperature 
Table A2. Excluded studies.

\begin{tabular}{|c|c|}
\hline Primary Author, Publication Year & Reason for Exclusion \\
\hline Aaron L. 2003 & Review study \\
\hline Arroll M. 2012 & Not intervention of interest \\
\hline Arroll MA. 2014 & Not eligible control group \\
\hline Benor D. 2017 & Not population of interest \\
\hline Bentler S. 2005 & Not population of interest \\
\hline Craske N. 2009 & Not population of interest \\
\hline Crawley E. 2017 & Not population of interest \\
\hline Deale A. 1997 & Not intervention of interest \\
\hline Deale A. 2001 & Not eligible control group \\
\hline Densham S. 2016 & Not population of interest \\
\hline Fjorback LO. 2012 & Not population of interest \\
\hline Fjorback LO. 2013 & Not population of interest \\
\hline Fjorback LO. 2013 & Not population of interest \\
\hline Guthlin C. 2012 & Not intervention of interest \\
\hline Hlavaty LE. 2011 & Not intervention of interest \\
\hline Hall DL. 2017 & Not eligible control group \\
\hline Jacobson HB. 2017 & Not population of interest \\
\hline James, L. 1996 & Case study \\
\hline Jason L. 2007 & Not intervention of interest \\
\hline Kos D. 2015 & Not eligible control group \\
\hline Lee J. 2015 & Not population of interest \\
\hline Nijs J. 2008 & Not intervention of interest \\
\hline Oka T. 2017 & Not eligible control group \\
\hline Pauzano-Slamm N. 2005 & Not peer-reviewed publication \\
\hline Ryan M. 2004 & Not population of interest \\
\hline Sampalli T. 2009 & Not population of interest \\
\hline Stevens MW. 1999 & Full-text not available \\
\hline Toussaint L. 2012 & Not population of interest \\
\hline Walach H. 2008 & Not intervention of interest \\
\hline Windthorst P. 2017 & Not eligible control group \\
\hline
\end{tabular}


Table A3. Statistically insignificant outcomes in the included studies using CDC, Canadian and international consensus criteria for diagnosing CFS.

\begin{tabular}{|c|c|c|c|c|c|c|}
\hline Intervention Type & First At & & Outcome (Assessed by) & Comparison Groups & Comparison Time Point & $p$-Value \\
\hline \multirow{3}{*}{ Relaxation-based } & \multirow{3}{*}{$\begin{array}{c}\text { Thomas, M., } 2006 \text { and } 2008 \\
{[39,51]}\end{array}$} & \multirow{2}{*}{ Report 1 (2006) } & $\begin{array}{l}\text { Anxiety (as part of a } \\
\text { self-report subjective mood } \\
\text { scale) }\end{array}$ & Relaxation group (pre-post) & Post treatment (10 weeks) & Non-significant \\
\hline & & & $\begin{array}{c}\text { Performance (word recall, } \\
\text { reaction time and vigilance } \\
\text { tasks) }\end{array}$ & Relaxation group (pre-post) & Post follow-up (6 months) & Non-significant \\
\hline & & Report 2 (2008) & $\begin{array}{c}\text { Performance score- } 10 \% \\
\text { improvement or } 80 \% \\
\text { attainment (Karnofsky scale) }\end{array}$ & $\begin{array}{l}\text { Relaxation group compared } \\
\text { to MCT and control groups }\end{array}$ & Post treatment (10 weeks) & Non-significant \\
\hline \multirow{9}{*}{ Cognitive-based } & \multirow{9}{*}{\multicolumn{2}{|c|}{ Sollie K., 2017 [43] (Mindfulness-based cognitive therapy) }} & $\begin{array}{c}\text { Fatigue (Profile of Mood } \\
\text { States (POMS) }\end{array}$ & CBSM compared to control & Time X group ** & 0.06 \\
\hline & & & $\begin{array}{l}\text { Fatigue (Chalder Fatigue } \\
\text { Scale) }\end{array}$ & MBCT (pre-post) & Post follow-up (3 months) & $\begin{array}{l}p \text { value not reported, } \\
\text { small effect size was } \\
\text { reported }(\mathrm{d}=0.26)\end{array}$ \\
\hline & & & Depression (HADS) & MBCT (pre-post) & Post intervention (8 weeks) & $\begin{array}{l}p \text { value not reported, } \\
\text { small effect size was } \\
\text { reported }(\mathrm{d}=0.32)\end{array}$ \\
\hline & & & Depression (HADS) & MBCT (pre-post) & Post follow-up (3 months) & $\begin{array}{l}p \text { value not reported, } \\
\text { small effect size was } \\
\text { reported }(\mathrm{d}=0.33)\end{array}$ \\
\hline & & & $\begin{array}{l}\text { Dispositional mindfulness } \\
\text { (Five Facet Mindfulness } \\
\text { questionnaire) }\end{array}$ & MBCT (pre-post) & Post intervention (8 weeks) & $\begin{array}{l}p \text { value not reported, } \\
\text { small effect size was } \\
\text { reported }(\mathrm{d}=0.11)\end{array}$ \\
\hline & & & $\begin{array}{l}\text { Rumination (Ruminative } \\
\text { Response Scale) }\end{array}$ & MBCT (pre-post) & Post intervention (8 weeks) & $\begin{array}{l}p \text { value not reported, } \\
\text { small effect size was } \\
\text { reported }(\mathrm{d}=0.26)\end{array}$ \\
\hline & & & $\begin{array}{l}\text { Rumination (Ruminative } \\
\text { Response Scale) }\end{array}$ & MBCT (pre-post) & Post follow-up (3 months) & $\begin{array}{l}p \text { value not reported, } \\
\text { small effect size was } \\
\text { reported }(\mathrm{d}=0.32)\end{array}$ \\
\hline & & & CFS symptom burden & MBCT (pre-post) & Post intervention (8 weeks) & $\begin{array}{l}p \text { value not reported, } \\
\text { small effect size was } \\
\text { reported }(\mathrm{d}=0.07)\end{array}$ \\
\hline & & & CFS symptom burden & MBCT (pre-post) & Post follow-up (3 months) & $\begin{array}{l}p \text { value not reported, } \\
\text { small effect size was } \\
\text { reported }(\mathrm{d}=0.04)\end{array}$ \\
\hline
\end{tabular}


Table A3. Cont.

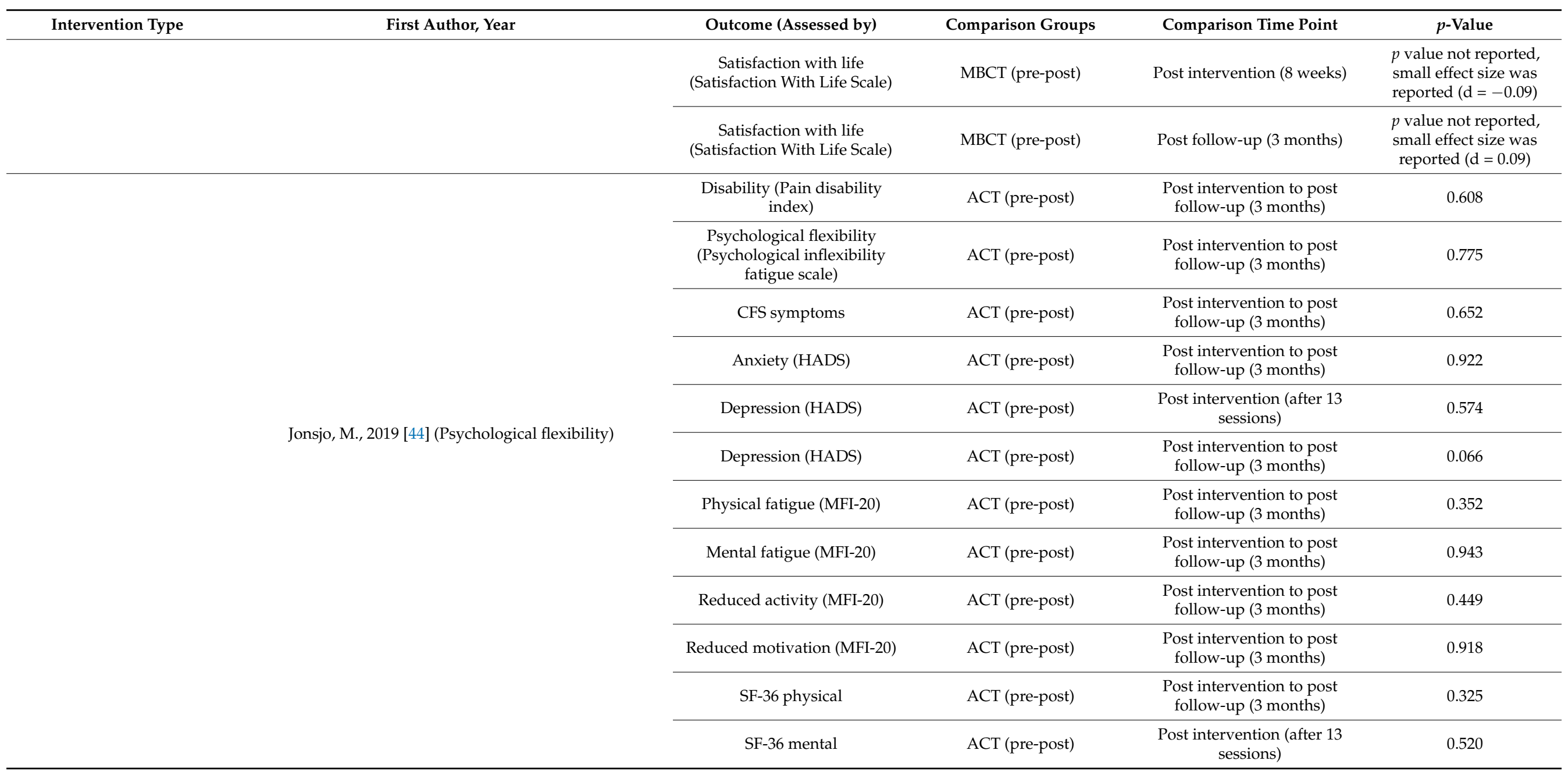


Table A3. Cont.

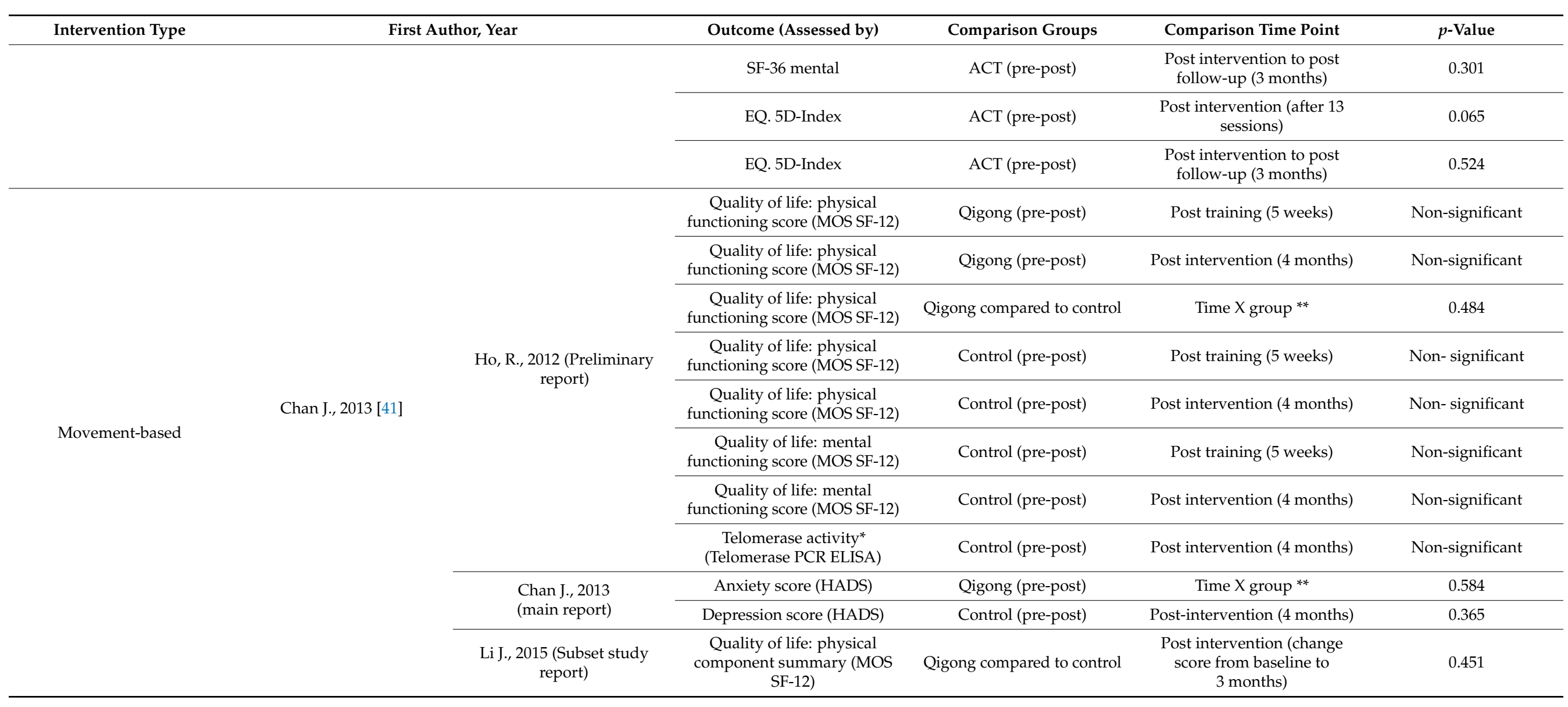


Table A3. Cont.

\begin{tabular}{|c|c|c|c|c|c|c|}
\hline \multirow[t]{11}{*}{ Intervention Type } & \multicolumn{2}{|c|}{ First Author, Year } & Outcome (Assessed by) & Comparison Groups & Comparison Time Point & $p$-Value \\
\hline & \multirow{6}{*}{$\begin{array}{c}\text { Chan, J. } 2014 \text { [40] and Chan, } \\
\text { J. } 2017 \text { [83] }\end{array}$} & \multirow{6}{*}{ Report 1 (2014) } & $\begin{array}{l}\text { Sleep quality: total score } \\
\text { (PSQI) }\end{array}$ & $\begin{array}{l}\text { Baduanjin Qigong } \\
\text { compared to waitlist }\end{array}$ & Time $\mathrm{X}$ group ${ }^{* *}$ & 0.064 \\
\hline & & & Sleep duration (PSQI) & $\begin{array}{l}\text { Baduanjin Qigong } \\
\text { compared to waitlist }\end{array}$ & Time $X$ group ** & 0.151 \\
\hline & & & Sleep efficacy (PSQI) & $\begin{array}{c}\text { Baduanjin Qigong } \\
\text { compared to wait list }\end{array}$ & Time X group ** & 0.522 \\
\hline & & & Sleep disturbance (PSQI) & $\begin{array}{l}\text { Baduanjin Qigong } \\
\text { compared to waitlist }\end{array}$ & Time X group ** & 0.062 \\
\hline & & & $\begin{array}{c}\text { Use of sleep medication } \\
\text { (PSQI) }\end{array}$ & $\begin{array}{l}\text { Baduanjin Qigong } \\
\text { compared to waitlist }\end{array}$ & Time X group ** & 0.803 \\
\hline & & & Daytime dysfunction (PSQI) & $\begin{array}{l}\text { Baduanjin Qigong } \\
\text { compared to waitlist }\end{array}$ & Time X group ** & 0.253 \\
\hline & & \multirow{4}{*}{ Report 2 (2017) } & Adiponectin levels & $\begin{array}{l}\text { Baduanjin Qigong } \\
\text { compared to waitlist }\end{array}$ & $\begin{array}{l}\text { Post intervention (change } \\
\text { score from baseline to } \\
\text { 3-month) }\end{array}$ & Non-significant \\
\hline & & & Depression (HADS) & $\begin{array}{l}\text { Baduanjin Qigong } \\
\text { compared to waitlist }\end{array}$ & $\begin{array}{l}\text { Post intervention (change } \\
\text { score from baseline to } \\
\text { 3-month) }\end{array}$ & Non-significant \\
\hline & & & Anxiety (HADS) & $\begin{array}{l}\text { Baduanjin Qigong } \\
\text { compared to waitlist }\end{array}$ & $\begin{array}{l}\text { Post intervention (change } \\
\text { score from baseline to } 9 \\
\text { weeks) }\end{array}$ & Non-significant \\
\hline & & & Anxiety (HADS) & $\begin{array}{l}\text { Baduanjin Qigong } \\
\text { compared to waitlist }\end{array}$ & $\begin{array}{l}\text { Post intervention (change } \\
\text { score from baseline to } \\
\text { 3-month) }\end{array}$ & Non-significant \\
\hline
\end{tabular}


Table A3. Cont.

\begin{tabular}{|c|c|c|c|c|c|c|}
\hline \multirow[t]{7}{*}{ Intervention Type } & \multicolumn{2}{|c|}{ First Author, Year } & \multirow{2}{*}{$\begin{array}{c}\text { Outcome (Assessed by) } \\
\text { Quality of life: vitality (SF-8) }\end{array}$} & \multirow{2}{*}{$\begin{array}{c}\text { Comparison Groups } \\
\text { Isometric yoga (pre-post) }\end{array}$} & \multirow{2}{*}{$\begin{array}{c}\text { Comparison Time Point } \\
\text { Post intervention (2 months) }\end{array}$} & \multirow{2}{*}{$\begin{array}{c}p \text {-Value } \\
\text { Non-significant }\end{array}$} \\
\hline & & & & & & \\
\hline & & & $\begin{array}{l}\text { Quality of life: role emotional } \\
\text { (SF-8) }\end{array}$ & Isometric yoga (pre-post) & Post intervention ( 2 months) & Non-significant \\
\hline & \multirow{4}{*}{\multicolumn{2}{|c|}{ Oka T, 2014 [28] }} & $\begin{array}{l}\text { Quality of life: physical } \\
\text { functioning (SF-8) }\end{array}$ & Isometric yoga (pre-post) & Post intervention ( 2 months) & Non-significant \\
\hline & & & $\begin{array}{l}\text { Quality of life: mental } \\
\text { component summary (SF-8) }\end{array}$ & Isometric yoga (pre-post) & Post intervention ( 2 months) & Non-significant \\
\hline & & & $\begin{array}{l}\text { Quality of life: role physical } \\
\text { (SF-8) }\end{array}$ & Isometric yoga (pre-post) & Post intervention ( 2 months) & Non-significant \\
\hline & & & $\begin{array}{l}\text { Quality of life: social } \\
\text { functioning (SF-8) }\end{array}$ & Isometric yoga (pre-post) & Post intervention ( 2 months) & Non-significant \\
\hline & \multirow{2}{*}{$\begin{array}{l}\text { Oka, T., } 2018 \text { and Oka, T., } \\
2019[42,91]\end{array}$} & \multirow{2}{*}{ Report 1 (2018) } & $\begin{array}{l}\text { Autonomic function indices } \\
\text { (low-frequency power of HR } \\
\text { variability, CVR-R: Coefficient } \\
\text { of variation of R-R intervals) }\end{array}$ & $\begin{array}{l}\text { Acute effects of sitting } \\
\text { isometric yoga (pre-post) }\end{array}$ & $\begin{array}{l}\text { Before to after the final 20-min } \\
\text { session }\end{array}$ & Non-significant \\
\hline & & & $\begin{array}{c}\text { Plasma biomarkers } \\
\text { (Transforming growth } \\
\text { factor-beta1; Brain-derived } \\
\text { Neurotrophic factor, } \\
\text { Homovanillic acid, } \\
\text { 3-methoxy-4- } \\
\text { hydroxyphenylglycol) }\end{array}$ & $\begin{array}{l}\text { Acute effects of sitting } \\
\text { isometric yoga (pre-post) }\end{array}$ & $\begin{array}{l}\text { Before to after the final 20-min } \\
\text { session }\end{array}$ & Non-significant \\
\hline
\end{tabular}


Table A3. Cont.

\begin{tabular}{|c|c|c|c|c|c|}
\hline Intervention Type & First Author, Year & Outcome (Assessed by) & Comparison Groups & Comparison Time Point & $p$-Value \\
\hline & \multirow{4}{*}{ Report 2 (2019) } & $\begin{array}{l}\text { Autonomic function tests, } \\
\text { serum, and blood biomarkers }\end{array}$ & $\begin{array}{l}\text { Longitudinal effects of } \\
\text { sitting isometric yoga } \\
\text { (pre-post) }\end{array}$ & Post intervention (2 months) & Non-significant \\
\hline & & Anxiety (HADS) & $\begin{array}{l}\text { Seated isometric yoga } \\
\text { compared to controls }\end{array}$ & Time $X$ group $* *$ & 0.786 \\
\hline & & Depression (HADS) & $\begin{array}{l}\text { Seated isometric yoga } \\
\text { compared to controls }\end{array}$ & Time $X$ group $* *$ & 0.008 \\
\hline & & Alexithymia (TAS-20) & $\begin{array}{l}\text { Seated isometric yoga } \\
\text { compared to controls }\end{array}$ & Time $X$ group ** & 0.950 \\
\hline
\end{tabular}

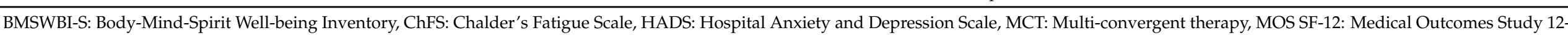

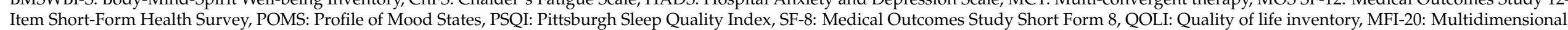

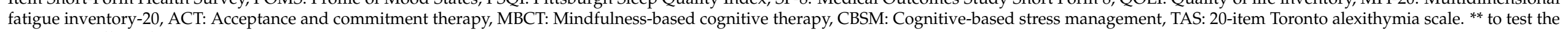
interaction effect of time and group.

Table A4. Statistically insignificant outcomes in the included studies using Oxford criteria for diagnosing CFS.

\begin{tabular}{|c|c|c|c|c|c|c|}
\hline Intervention Type & First $\mathrm{A}$ & Year & Outcome (Assessed by) & Comparison Groups & Comparison Time Point & $p$-Value \\
\hline \multirow{5}{*}{$\begin{array}{l}\text { Mindfulness and } \\
\text { cognitive-based }\end{array}$} & \multirow{5}{*}{ Surawy, Ch., 2005 [29] } & \multirow{2}{*}{ Study 1 (RCT) } & Depression (HADS) & $\begin{array}{l}\text { MBSR/MBCT compared to } \\
\text { controls }\end{array}$ & Post treatment (8 weeks) & 0.28 \\
\hline & & & Fatigue score (ChFS) & $\begin{array}{l}\text { MBSR/MBCT compared to } \\
\text { controls }\end{array}$ & Post treatment (8 weeks) & 0.08 \\
\hline & & \multirow{3}{*}{$\begin{array}{l}\text { Study } 2 \text { (single-arm } \\
\text { experimental study) }\end{array}$} & Depression (HADS) & MBCT/MBSR (pre-post) & Post treatment (8 weeks) & 0.16 \\
\hline & & & Fatigue score (ChFS) & MBCT/MBSR (pre-post) & Post treatment (8 weeks) & 0.06 \\
\hline & & & $\begin{array}{c}\text { Quality of life: physical functioning } \\
\text { (SF-36) }\end{array}$ & MBCT/MBSR (pre-post) & Post treatment (8 weeks) & 0.69 \\
\hline
\end{tabular}


Table A4. Cont.

Outcome (Assessed by)

Comparison Groups

Comparison Time Point

$p$-Value

Mindfulness (5 facet mindfulness questionnaire)

\begin{tabular}{clcc}
\hline questionnaire) & & & 0.152 \\
\hline Catastrophizing (Self-reporting scale) & MBCT compared to waitlist & Post follow-up (2 months) & 0.089 \\
\hline $\begin{array}{c}\text { All-or-nothing behavior } \\
\text { (Self-reporting scale) }\end{array}$ & MBCT compared to waitlist & Post follow-up (2 months) & 0.153 \\
\hline Depression (HADS) & MBCT compared to waitlist & Post follow-up (2 months) & 0.173 \\
\hline Anxiety (HADS) & MBCT compared to waitlist & Post treatment (8 weeks) & 0.296 \\
\hline Anxiety (HADS) & MBCT compared to waitlist & Post follow-up (2 months) & 0.124 \\
\hline $\begin{array}{c}\text { Quality of life: physical functioning } \\
\text { (SF-36) }\end{array}$ & MBCT compared to waitlist & Post treatment (8 weeks) & 0.345 \\
\hline $\begin{array}{c}\text { Quality of life: physical functioning } \\
\text { (SF-36) }\end{array}$ & MBCT compared to waitlist & Post follow-up (2 months) & 0.054
\end{tabular}

Rimes, K., 2013 [30]

\begin{tabular}{cccc}
\hline $\begin{array}{c}\text { Impairment (The work and social } \\
\text { adjustment scale) }\end{array}$ & MBCT compared to waitlist & Post follow-up (2 months) & 0.054 \\
\hline Fatigue (ChFS) & MBCT (pre-post) & Between 2- and 6-month follow-up & 0.089 \\
\hline Depression (HADS) & MBCT (pre-post) & Between 2- and 6-month follow-up & 0.069 \\
\hline $\begin{array}{c}\text { Catastrophizing (Self-reporting scale) } \\
\begin{array}{c}\text { All-or-nothing behavior } \\
\text { (Self-reporting scale) }\end{array}\end{array}$ MBCT (pre-post) & Between 2- and 6-month follow-up & 0.063 \\
\hline $\begin{array}{c}\text { Self-Compassion (Self-reporting scale) } \\
\text { Anxiety (HADS) }\end{array}$ & MBCT (pre-post) & Between 2- and 6-month follow-up & 0.082 \\
\hline $\begin{array}{c}\text { Quality of life: physical functioning } \\
\text { (SF-36) }\end{array}$ & MBCT (pre-post) & Between 2- and 6-month follow-up & 0.110 \\
\hline $\begin{array}{c}\text { Beliefs about Emotions (Self-reporting } \\
\text { scale) }\end{array}$ & MBCT (pre-post) & Between 2- and 6-month follow-up & 0.84 \\
\hline $\begin{array}{c}\text { Quality of life: physical functioning } \\
\text { (SF-36) }\end{array}$ & MBCT (pre-post) & Post follow-up (6 months) & 0.051 \\
\hline Depression (HADS) & MBCT (pre-post) & Post follow-up (6 months) & 0.051 \\
\hline Anxiety (HADS) & MBCT (pre-post) & Post follow-up (6 months) & 0.206 \\
\hline
\end{tabular}

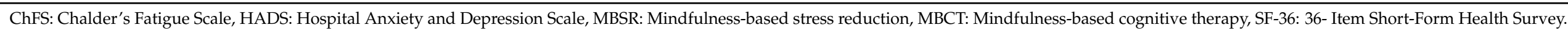


Table A5. Risk of Bias (ROBINS-I).

\begin{tabular}{|c|c|c|c|c|c|c|c|}
\hline Domains & $\begin{array}{l}\text { Bogaerts } \\
2007\end{array}$ & $\begin{array}{l}\text { Surawy } 2005 \\
\text { Study } 2\end{array}$ & $\begin{array}{l}\text { Surawy } 2005 \\
\text { Study } 3\end{array}$ & Sollie 2017 & $\begin{array}{l}\text { Oka } 2018 \\
\text { and } 2019\end{array}$ & Jonsjo 2019 & $\begin{array}{l}\text { Takakura } \\
2019\end{array}$ \\
\hline Confounding & $\begin{array}{l}\text { No } \\
\text { information }\end{array}$ & $\begin{array}{l}\text { No } \\
\text { information }\end{array}$ & $\begin{array}{l}\text { No } \\
\text { information }\end{array}$ & Low & Low & Low & Low \\
\hline Selection bias & Low & Serious & Serious & Low & Moderate & Low & Low \\
\hline $\begin{array}{l}\text { Measurement } \\
\text { of } \\
\text { intervention }\end{array}$ & Low & Low & Low & Low & Low & Low & Low \\
\hline $\begin{array}{l}\text { Deviation } \\
\text { from the } \\
\text { intended } \\
\text { intervention }\end{array}$ & Low & Low & Low & Low & Low & Low & Low \\
\hline Missing data & Low & Low & Low & Low & Low & Low & Low \\
\hline $\begin{array}{l}\text { Measurement } \\
\text { of outcomes }\end{array}$ & Moderate & Serious & Serious & Moderate & Moderate & Moderate & Moderate \\
\hline $\begin{array}{l}\text { Reported } \\
\text { results }\end{array}$ & $\begin{array}{l}\text { No } \\
\text { information }\end{array}$ & $\begin{array}{l}\text { No } \\
\text { information }\end{array}$ & $\begin{array}{l}\text { No } \\
\text { information }\end{array}$ & Low & Low & Low & Low \\
\hline Overall & Moderate & Serious & Serious & Moderate & Moderate & Moderate & Moderate \\
\hline
\end{tabular}

Low risk of bias (the study is comparable to a well-performed randomized trial with regard to this domain), Moderate risk of bias (the study is sound for a nonrandomized study with regard to this domain but cannot be considered comparable to a well-performed randomized trial), Serious risk of bias (the study has some important problems).

Table A6. Brief descriptions of mind-body interventions used in the included studies.

\begin{tabular}{|c|c|}
\hline MBIs & Definition \\
\hline Relaxation therapies & $\begin{array}{l}\text { https://www.nccih.nih.gov/health/relaxation-techniques-for-health (accessed on } \\
13 \text { June 2021). } \\
\text { "Relaxation techniques include a number of practices such as progressive } \\
\text { relaxation, guided imagery, biofeedback, self-hypnosis, and deep breathing } \\
\text { exercises. The goal is similar in all: to produce the body's natural relaxation } \\
\text { response, characterized by slower breathing, lower blood pressure, and a feeling of } \\
\text { increased well-being". }\end{array}$ \\
\hline \multirow[t]{2}{*}{ Movement-based interventions } & $\begin{array}{l}\text { https://www.nccih.nih.gov/health/yoga-what-you-need-to-know (accessed on } \\
13 \text { June 2021). } \\
\text { "Although classical yoga also includes other elements, yoga as practiced in the } \\
\text { United States typically emphasizes physical postures (asanas), breathing } \\
\text { techniques (pranayama), and meditation (dyana). } \\
\text { There are many different yoga styles, ranging from gentle practices to physically } \\
\text { demanding ones. Differences in the types of yoga used in research studies may } \\
\text { affect study results. This makes it challenging to evaluate research on the health } \\
\text { effects of yoga. } \\
\text { Yoga and two practices of Chinese origin-tai chi and qi gong-are sometimes } \\
\text { called "meditative movement" practices. All three practices include both } \\
\text { meditative elements and physical ones". }\end{array}$ \\
\hline & $\begin{array}{l}\text { https:/ / www.nccih.nih.gov/health/tai-chi-and-qi-gong-in-depth (accessed on } 13 \\
\text { June 2021). } \\
\text { "Tai chi and qi gong are centuries-old practices that involve certain postures and } \\
\text { gentle movements with mental focus, breathing, and relaxation. The movements } \\
\text { can be adapted or practiced while walking, standing, or sitting. In contrast to qi } \\
\text { gong, tai chi movements, if practiced quickly, can be a form of combat or } \\
\text { self-defense". }\end{array}$ \\
\hline
\end{tabular}


Table A6. Cont.

\begin{tabular}{|c|c|}
\hline MBIs & Definition \\
\hline \multirow{4}{*}{ Mindfulness and cognitive-based } & $\begin{array}{l}\text { Mindfulness-based stress reduction (MBSR): } \\
\text { "The program is conducted as an } 8-\text { to } 10 \text {-week course for groups of up to } 30 \\
\text { participants who meet weekly for } 2-2.5 \mathrm{hr} \text { for instruction and practice in } \\
\text { mindfulness meditation skills, together with a discussion of stress, coping, and } \\
\text { homework assignments". } 1\end{array}$ \\
\hline & $\begin{array}{l}\text { Mindfulness-based cognitive therapy (MBCT): } \\
\text { "MBCT incorporates elements of cognitive therapy that facilitate a detached or } \\
\text { de-centered view of one's thoughts, including statements such as "thoughts are } \\
\text { not facts" and "I am not my thoughts." This decentered approach also is applied to } \\
\text { emotions and bodily sensations". } 1\end{array}$ \\
\hline & $\begin{array}{l}\text { Cognitive-behavioral stress management (CBSM) is based on cognitive } \\
\text { restructuring: "CBSM interventions reduce distress by teaching relaxation } \\
\text { techniques; modifying patients' outlook, cognitive appraisals, and coping } \\
\text { strategies; and when performed in } \\
\text { a group format may also improve their perceptions of social support". } 2\end{array}$ \\
\hline & $\begin{array}{l}\text { Acceptance commitment therapy }(\mathrm{ACT}) \text { is based on psychological flexibility. } \\
\text { "This is defined as the ability to act in line with important long-term goals or } \\
\text { values in life, even in the presence of negative experiences (e.g., non-acute somatic } \\
\text { symptoms or psychological distress). Psychological flexibility is a complex } \\
\text { overarching behavioral construct that includes several behavioral processes such } \\
\text { as acceptance/non-acceptance and cognitive fusion/diffusion". } 3\end{array}$ \\
\hline
\end{tabular}

\begin{abstract}
${ }^{1}$ Baer RA. Mindfulness training as a clinical intervention: A conceptual and empirical review. Clinical psychology: Science and practice. June 2003, 10,125-143. ${ }^{2}$ Lopez C, Antoni M, Penedo F, Weiss D, Cruess S, Segotas M-C, et al. A pilot study of cognitive-behavioral stress management effects on stress, quality of life, and symptoms in persons with chronic fatigue syndrome. Journal of psychosomatic research. 2011, 70, 328-334. ${ }^{3}$ Jonsjö MA, Wicksell RK, Holmström L, Andreasson A, Olsson GL. Acceptance and commitment therapy for ME/CFS (Chronic Fatigue Syndrome)-a feasibility study. Journal of Contextual Behavioral Science. 2019, $12,89-97$.
\end{abstract}

\title{
Appendix B
}

\section{Medline Search Strategy}

1. Fatigue Syndrome, Chronic/

2. Myalgic Encephalomyelitis.mp.

3. exp Encephalomyelitis/

4. Fatigue/

5. 3 and 4

6. 1 or 2 or 5

7. (chronic $\$$ adj3 fatig $\$$ adj3 syndrom\$).mp.

8. (myalg $\$$ adj3 encephal\$).mp.

9. 6 or 7 or 8

10. exp Mind-Body Therapies/

11. exp Biofeedback, Psychology/

12. exp Neurofeedback/

13. exp "Imagery (Psychotherapy)" /

14. exp Hypnosis/

15. $\exp$ Relaxation Therapy/

16. exp Mindfulness/

17. exp Meditation/

18. exp Yoga/

19. $\exp$ Tai Ji/

20. (Mindfulness-based adj2 cognitive adj2 therapy).mp.

21. self-hypnosis.mp.

22. Guided imagery.mp.

23. exp Art Therapy/

24. mindfulness-based stress reduction.mp. 


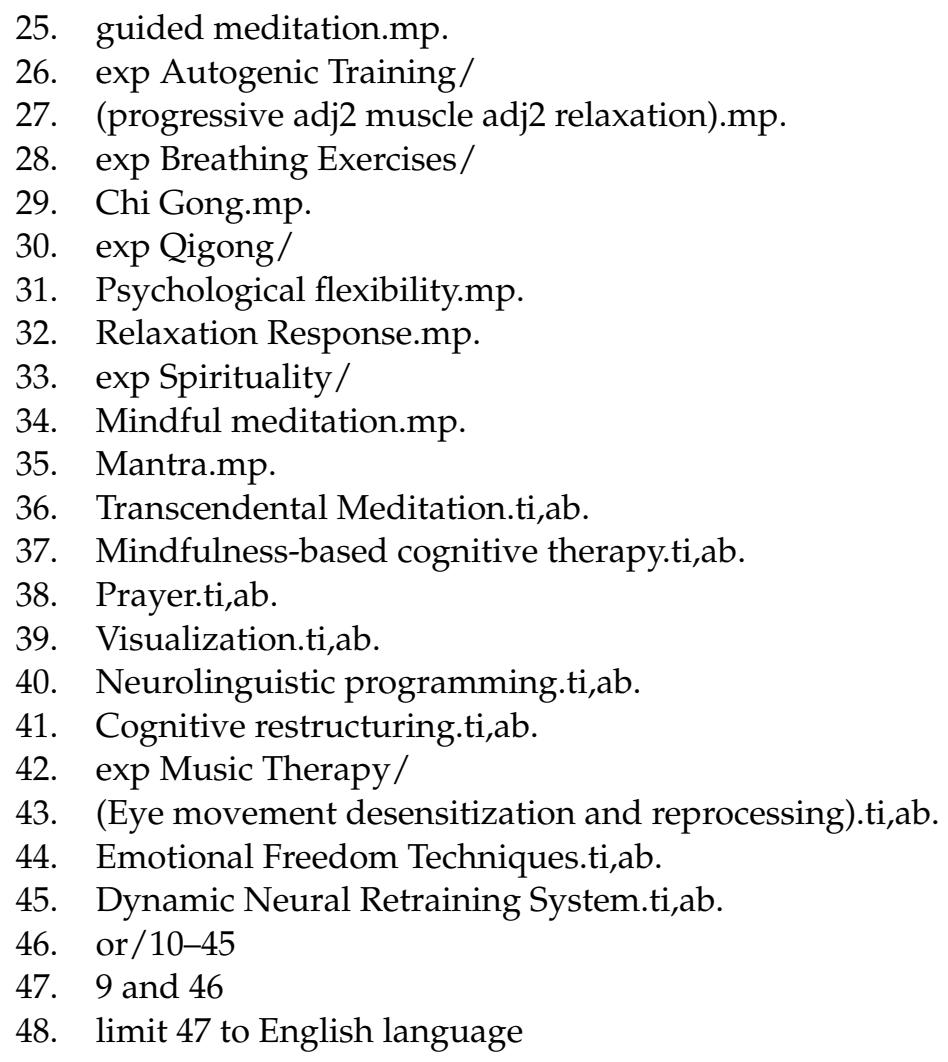

\section{References}

1. Institute of Medicine. Beyond Myalgic Encephalomyelitis/Chronic Fatigue Syndrome: Redefining an Illness, Committe on the Diagnosis for ME/CFS.; The National Academies Pres: Washington, DC, USA, 2015.

2. Johnston, S.; Brenu, E.W.; Staines, D.; Marshall-Gradisnik, S. The prevalence of chronic fatigue syndrome/myalgic encephalomyelitis: A meta-analysis. Clin. Epidemiol. 2013, 5, 105-110. [CrossRef] [PubMed]

3. Rusu, C.; Gee, M.E.; Lagacé, C.; Parlor, M. Chronic fatigue syndrome and fibromyalgia in Canada: Prevalence and associations with six health status indicators. Chronic Dis. Inj. Can. 2015, 35, 1678508400. [CrossRef]

4. Jason, L.A.; Richman, J.A.; Rademaker, A.W.; Jordan, K.M.; Plioplys, A.V.; Taylor, R.R.; McCready, W.; Huang, C.F.; Plioplys, S. A community-based study of chronic fatigue syndrome. Arch. Intern. Med. 1999, 159, 2129-2137. [CrossRef] [PubMed]

5. Hvidberg, M.F.; Brinth, L.S.; Olesen, A.V.; Petersen, K.D.; Ehlers, L. The Health-Related Quality of Life for Patients with Myalgic Encephalomyelitis/Chronic Fatigue Syndrome (ME/CFS). PLoS ONE 2015, 10, e0132421. [CrossRef]

6. Nacul, L.C.; Lacerda, E.M.; Campion, P.; Pheby, D.; Drachler, M.d.L.; Leite, J.C.; Poland, F.; Howe, A.; Fayyaz, S.; Molokhia, M. The functional status and well being of people with myalgic encephalomyelitis/chronic fatigue syndrome and their carers. $B M C$ Public Health 2011, 11, 402. [CrossRef] [PubMed]

7. Bombardier, C.H.; Buchwald, D. Chronic fatigue, chronic fatigue syndrome, and fibromyalgia. Disability and health-care use. Med. Care 1996, 34, 924-930. [CrossRef] [PubMed]

8. Reynolds, K.J.; Vernon, S.D.; Bouchery, E.; Reeves, W.C. The economic impact of chronic fatigue syndrome. Cost Eff. Resour. Alloc. 2004, 2, 1-4. [CrossRef]

9. Centers for Diseases Control and Prevention. Chronic Fatigue Syndrome Awareness Day: CDC. 2018. Available online: http:/ / www.cdc.gov/Features/cfsawarenessday/ (accessed on 21 August 2018).

10. Takakura, S.; Oka, T.; Sudo, N. Changes in circulating microRNA after recumbent isometric yoga practice by patients with myalgic encephalomyelitis/chronic fatigue syndrome: An explorative pilot study. Bio. Psycho. Soc. Med. 2019, 13, 1-10. [CrossRef] [PubMed]

11. Missailidis, D.; Annesley, S.J.; Fisher, P.R. Pathological mechanisms underlying myalgic encephalomyelitis/chronic fatigue syndrome. Diagnostics 2019, 9, 80. [CrossRef] [PubMed]

12. Maksoud, R.; du Preez, S.; Eaton-Fitch, N.; Thapaliya, K.; Barnden, L.; Cabanas, H.; Staines, D.; Marshall-Gradisnik, S. A systematic review of neurological impairments in myalgic encephalomyelitis/chronic fatigue syndrome using neuroimaging techniques. PLoS ONE 2020, 15, e0232475. [CrossRef] 
13. Haney, E.; Smith, M.E.B.; McDonagh, M.; Pappas, M.; Daeges, M.; Wasson, N.; Nelson, H.D. Diagnostic Methods for Myalgic Encephalomyelitis/Chronic Fatigue Syndrome: A Systematic Review for a National Institutes of Health Pathways to Prevention WorkshopDiagnostic Methods for Myalgic Encephalomyelitis/Chronic Fatigue Syndrome. Ann. Intern. Med. 2015, 162, 834-840. [CrossRef]

14. Smith, M.; Nelson, H.D.; Haney, E.; Pappas, M.; Daeges, M.; Wasson, N.; McDonagh, M. Diagnosis and Treatment of Myalgic Encephalomyelitis/Chronic Fatigue Syndrome. Agency for Healthcare Research and Quality Evidence Report/Technology Assessment No 219 (Prepared by the Pacific Northwest Evidence-based Practice Center under Contract No 290-2012-00014-I) Rockville, MD: AHRQ Publication No. 15-E001-EF. Available online: www.effectivehealthcare.ahrq.gov/reports/final.cfm (accessed on 20 June 2021).

15. Holgate, S.T.; Komaroff, A.L.; Mangan, D.; Wessely, S. Chronic fatigue syndrome: Understanding a complex illness. Nat. Rev. Neurosci. 2011, 12, 539-544. [CrossRef] [PubMed]

16. Acheson, E.D. The clinical syndrome variously called benign myalgic encephalomyelitis, Iceland disease and epidemic neuromyasthenia. Am. J. Med. 1959, 26, 569-595. [CrossRef]

17. Holmes, G.P.; Kaplan, J.E.; Gantz, N.M.; Komaroff, A.L.; Schonberger, L.B.; Straus, S.E.; Jones, J.F.; Dubois, R.E.; CunninghamRundles, C.; Pahwa, S.; et al. Chronic Fatigue Syndrome: A Working Case Definition. Ann. Intern. Med. 1988, 108, 387-389. [CrossRef] [PubMed]

18. Jason, L.A.; Brown, A.; Evans, M.; Sunnquist, M.; Newton, J.L. Contrasting Chronic Fatigue Syndrome versus Myalgic Encephalomyelitis/Chronic Fatigue Syndrome. Fatigue: Biomedicine, health \& behavior. Ann. Intern. Med. 2013, 1, 168-183. [CrossRef]

19. Smith, M.E.B.; Haney, E.; McDonagh, M.; Pappas, M.; Daeges, M.; Wasson, N.; Fu, R.; Nelson, H.D. Treatment of Myalgic Encephalomyelitis/Chronic Fatigue Syndrome: A Systematic Review for a National Institutes of Health Pathways to Prevention WorkshopTreatment of Myalgic Encephalomyelitis/Chronic Fatigue Syndrome. Ann. Intern. Med. 2015, 162, 841-850. [CrossRef]

20. Toward Optimal Practice. Identification and Symptom Management of ME/CFS.; Clinical Practice Guideline: Calgary, AB, Canada, 2016.

21. McCrone, P.; Darbishire, L.; Ridsdale, L.; Seed, P. The economic cost of chronic fatigue and chronic fatigue syndrome in UK primary care. Psychol. Med. 2003, 33, 253-261. [CrossRef] [PubMed]

22. Price, J.R.; Mitchell, E.; Tidy, E.; Hunot, V. Cognitive behaviour therapy for chronic fatigue syndrome in adults. Cochrane Database Syst. Rev. 2008. [CrossRef]

23. Larun, L.; Brurberg, K.G.; Odgaard-Jensen, J.; Price, J.R. Exercise therapy for chronic fatigue syndrome. Cochrane Database Syst. Rev. 2015. [CrossRef]

24. Wahbeh, H.; Elsas, S.-M.; Oken, B.S. Mind-body interventions: Applications in neurology. Neurology 2008, 70, 2321-2328. [CrossRef] [PubMed]

25. Gupta, A. Can amygdala retraining techniques improve the wellbeing of patients with chronic fatigue syndrome. J. Holist. Healthc. 2010, 7, 12-15.

26. Vitetta, L.; Anton, B.; Cortizo, F.; Sali, A. Mind-body medicine: Stress and its impact on overall health and longevity. Ann. N. Y. Acad. Sci. 2005, 1057, 492-505. [CrossRef]

27. Theadom, A.; Cropley, M.; Smith, H.E.; Feigin, V.L.; McPherson, K. Mind and body therapy for fibromyalgia. Cochrane Database Syst. Rev. 2015, 1057, 492-505. [CrossRef] [PubMed]

28. Oka, T.; Tanahashi, T.; Chijiwa, T.; Lkhagvasuren, B.; Sudo, N.; Oka, K. Isometric yoga improves the fatigue and pain of patients with chronic fatigue syndrome who are resistant to conventional therapy: A randomized, controlled trial. BioPsychoSocial Med. 2014, 8, 1-9. [CrossRef]

29. Surawy, C.; Roberts, J.; Silver, A. The Effect of Mindfulness Training on Mood and Measures of Fatigue, Activity, and Quality of Life in Patients with Chronic Fatigue Syndrome on a Hospital Waiting List: A Series of Exploratory Studies. Behav. Cogn. Psychother. 2005, 33, 103-109. [CrossRef]

30. Rimes, K.A.; Wingrove, J. Mindfulness-Based Cognitive Therapy for People with Chronic Fatigue Syndrome Still Experiencing Excessive Fatigue after Cognitive Behaviour Therapy: A Pilot Randomized Study. Clin. Psychol. Psychother. 2013, 20, 107-117. [CrossRef] [PubMed]

31. Collinge, W.; Yarnold, P.R.; Raskin, E. Use of mind/body selfhealing practice predicts positive health transition in chronic fatigue syndrome: A controlled study. Subtle Energ. Energy Med. J. Arch. 1998, 9, 107-117.

32. Dybwad, M.; Frøslie, K.; Stanghelle, J. Work Capacity, Fatigue and Health Related Quality of Life in Patients with Myalgic Encephalopathy or Chronic Fatigue Syndrome, before and after Qigong Therapy, a Randomized Controlled Study; Sunnaas Rehabilitation Hospital: Nesoddtangen, Norway, 2007.

33. Moher, D.; Hopewell, S.; Schulz, K.F.; Montori, V.; Gotzsche, P.C.; Devereaux, P.J.; Elbourne, D.; Egger, M.; Altman, D.G.; Consolidated Standards of Reporting Trials Group. CONSORT 2010 Explanation and Elaboration: Updated guidelines for reporting parallel group randomised trials. J. Clin. Epidemiol. 2010, 63, e1-37. [CrossRef] [PubMed]

34. Higgins, J.P.T.; Altman, D.G.; Gøtzsche, P.C.; Jüni, P.; Moher, D.; Oxman, A.D.; Savović, J.; Schulz, K.F.; Weeks, L.; Sterne, J.A. The Cochrane Collaboration's tool for assessing risk of bias in randomised trials. BMJ 2011, 343, d5928. [CrossRef] 
35. Sterne, J.A.; Hernán, M.A.; Reeves, B.C.; Savović, J.; Berkman, N.D.; Viswanathan, M.; Henry, D.; Altman, D.G.; Ansari, M.T.; Boutron, I.; et al. ROBINS-I: A tool for assessing risk of bias in non-randomised studies of interventions. BMJ 2016, 355 , i4919. [CrossRef]

36. Domecq, J.P.; Prutsky, G.; Elraiyah, T.; Wang, Z.; Nabhan, M.; Shippee, N.; Brito, J.P.; Boehmer, K.; Hasan, R.; Firwana, B.; et al. Patient engagement in research: A systematic review. BMC Health Serv. Res. 2014, 14, 89. [CrossRef]

37. Pollock, A.; Campbell, P.; Struthers, C.; Synnot, A.; Nunn, J.; Hill, S.; Goodare, H.; Morris, J.; Watts, C.; Morley, R. Stakeholder involvement in systematic reviews: A scoping review. Syst. Rev. 2018, 7, 208. [CrossRef] [PubMed]

38. Bogaerts, K.; Hubin, M.; Van Diest, I.; De Peuter, S.; Van Houdenhove, B.; Van Wambeke, P.; Crombez, G.; Van den Bergh, O. Hyperventilation in patients with chronic fatigue syndrome: The role of coping strategies. Behav. Res. Ther. 2007, 45, 2679-2690. [CrossRef] [PubMed]

39. Thomas, M.A.; Sadlier, M.J.; Smith, A.P. A multiconvergent approach to the rehabilitation of patients with chronic fatigue syndrome: A comparative study. Physiotherap 2008, 94, 35-42. [CrossRef]

40. Chan, J.S.; Ho, R.T.; Chung, K.-F.; Wang, C.-W.; Yao, T.-J.; Ng, S.-M.; Chan, C.L. Qigong exercise alleviates fatigue, anxiety, and depressive symptoms, improves sleep quality, and shortens sleep latency in persons with chronic fatigue syndrome-like illness. Evid. Based Complementary Altern. Med. 2014, 2014, 106048. [CrossRef]

41. Chan, J.S.; Ho, R.T.; Wang, C.-W.; Yuen, L.P.; Sham, J.S.; Chan, C.L. Effects of qigong exercise on fatigue, anxiety, and depressive symptoms of patients with chronic fatigue syndrome-like illness: A randomized controlled trial. Evid. Based Complementary Altern. Med. 2013, 2013, 1-8. [CrossRef]

42. Oka, T.; Tanahashi, T.; Sudo, N.; Lkhagvasuren, B.; Yamada, Y. Changes in fatigue, autonomic functions, and blood biomarkers due to sitting isometric yoga in patients with chronic fatigue syndrome. BioPsychoSocial Med. 2018, 12, 3. [CrossRef] [PubMed]

43. Sollie, K.; Næss, E.T.; Solhaug, I.; Thimm, J. Mindfulness training for chronic fatigue syndrome: A pilot study. Health Psychol. Rep. 2017, 3, 240-250. [CrossRef]

44. Jonsjö, M.A.; Wicksell, R.K.; Holmström, L.; Andreasson, A.; Olsson, G.L. Acceptance \& commitment therapy for ME/CFS (Chronic Fatigue Syndrome)-a feasibility study. J. Contextual Behav. Sci. 2019, 12, 89-97.

45. Lopez, C.; Antoni, M.; Penedo, F.; Weiss, D.; Cruess, S.; Segotas, M.-C.; Helder, L.; Siegel, S.; Klimas, N.; Fletcher, M.A. A pilot study of cognitive behavioral stress management effects on stress, quality of life, and symptoms in persons with chronic fatigue syndrome. J. Psychosom. Res. 2011, 70, 328-334. [CrossRef] [PubMed]

46. Sharpe, M.C.; Archard, L.C.; Banatvala, J.E.; Borysiewicz, L.K.; Clare, A.W.; David, A.; Edwards, R.H.; Hawton, K.E.; Lambert, H.P.; Lane, R.J. A report—Chronic fatigue syndrome: Guidelines for research. J. R. Soc. Med. 1991, 84, 118-121. [CrossRef]

47. Zigmond, A.S.; Snaith, R.P. The hospital anxiety and depression scale. Acta Psychiatr. Scand. 1983, 67, 361-370. [CrossRef] [PubMed]

48. Chalder, T.; Berelowitz, G.; Pawlikowska, T.; Watts, L.; Wessely, S.; Wright, D.; Wallace, E.P. Development of a fatigue scale. J. Psychosom. Res. 1993, 37. [CrossRef]

49. Ware, J.E., Jr.; Sherbourne, C.D. The MOS 36-item short-form health survey (SF-36): I. Conceptual framework and item selection. Med. Care 1992, 30, 473-483. [CrossRef] [PubMed]

50. Fisk, J.D.; Ritvo, P.G.; Ross, L.; Haase, D.A.; Marrie, T.J.; Schlech, W.F. Measuring the functional impact of fatigue: Initial validation of the fatigue impact scale. Clin. Infect. Dis. 1994, 18 (Suppl. 1), S79-S83. [CrossRef]

51. Thomas, M.; Sadlier, M.; Smith, A. The effect of Multi Convergent Therapy on the psychopathology, mood and performance of Chronic Fatigue Syndrome patients: A preliminary study. Couns. Psychother. Res. 2006, 6, 91-99. [CrossRef]

52. Fukuda, K.; Straus, S.E.; Hickie, I.; Sharpe, M.C.; Dobbins, J.G.; Komaroff, A. The Chronic Fatigue Syndrome: A Comprehensive Approach to Its Definition and Study. Ann. Intern. Med. 1994, 121, 953-959. [CrossRef] [PubMed]

53. Beck, A.T.; Ward, C.H.; Mendelson, M.; Mock, J.; Erbaugh, J. An inventory for measuring depression. Arch. Gen. Psychiatry 1961, 4, 561-571. [CrossRef]

54. Radloff, L. The center for epidemiologic studies depression index. Appl. Psychol. Meas. 1977, 1, 385-401. [CrossRef]

55. Broadbent, D.E.; Cooper, P.F.; FitzGerald, P.; Parkes, K.R. The cognitive failures questionnaire (CFQ) and its correlates. Br. J. Clin. Psychol. 1982, 21, 1-16. [CrossRef] [PubMed]

56. Cohen, S.; Hoberman, H.M. Positive events and social supports as buffers of life change stress1. J. Appl. Soc. Psychol. 1983, 13, 99-125. [CrossRef]

57. Smith, A.; Pollock, J.; Thomas, M.; Llewelyn, M.; Borysiewicz, L. The relationship between subjective ratings of sleep and mental functioning in healthy subjects and patients with chronic fatigue syndrome. Hum. Psychopharmacol. Clin. Exp. 1996, 11, 161-167. [CrossRef]

58. Marks, I.M. Behavioural Psychotherapy: Maudsley Pocket Book of Clinical Management; Wright/IOP Publishing: Bristol, UK, 1986.

59. Zevon, M.A.; Tellegen, A. The structure of mood change: An idiographic/nomothetic analysis. J. Personal. Soc. Psychol. 1982, 43, 111. [CrossRef]

60. Ray, C.; Weir, W.; Stewart, D.; Miller, P.; Hyde, G. Ways of coping with chronic fatigue syndrome: Development of an illness management questionnaire. Soc. Sci. Med. 1993, 37, 385-391. [CrossRef]

61. Karnofsky, D.; Abelmann, W.; Craver, L. The use of the nitrogen mustards in the palliative treatment of carcinoma. With particular reference to bronchogenic carcinoma. Cancer 1948, 1, 634-656. [CrossRef] 
62. Watson, D.; Clark, L.A.; Tellegen, A. Development and validation of brief measures of positive and negative affect: The PANAS scales. J. Personal. Soc. Psychol. 1988, 54, 1063. [CrossRef]

63. Wientjes, C.J.; Grossman, P. Overreactivity of the psyche or the soma? Interindividual associations between psychosomatic symptoms, anxiety, heart rate, and end-tidal partial carbon dioxide pressure. Psychosom. Med. 1994, 56, 533-540. [CrossRef]

64. Meyer, T.J.; Miller, M.L.; Metzger, R.L.; Borkovec, T.D. Development and validation of the penn state worry questionnaire. Behav. Res. Ther. 1990, 28, 487-495. [CrossRef]

65. Bradley, M.M.; Lang, P.J. Measuring emotion: The self-assessment manikin and the semantic differential. J. Behav. Ther. Exp. Psychiatry 1994, 25, 49-59. [CrossRef]

66. Cohen, S.; Kamarck, T.; Mermelstein, R. A global measure of perceived stress. J. Health Soc. Behav. 1983, 24, 385-396. [CrossRef]

67. McNair, D.; Lorr, M.; DroppLemn, L. Manual for the Profile of Mood States (POMS); Educational and Industrial Testing Service: San Diego, CA, USA, 1971.

68. Frisch, M.B. Quality of Life Inventory (QOLI); National Computer Systems: Minneapolis, MN, USA, 1994.

69. Wagner, D.; Nisenbaum, R.; Heim, C.; Jones, J.F.; Unger, E.R.; Reeves, W.C. Psychometric properties of the CDC symptom inventory for assessment of Chronic Fatigue Syndrome. Popul. Health Metr. 2005, 3. [CrossRef] [PubMed]

70. Ho, R.T.; Chan, J.S.; Wang, C.-W.; Lau, B.W.; So, K.F.; Yuen, L.P.; Sham, J.S.; Chan, C.L. A randomized controlled trial of qigong exercise on fatigue symptoms, functioning, and telomerase activity in persons with chronic fatigue or chronic fatigue syndrome. Ann. Behav. Med. 2012, 44, 160-170. [CrossRef]

71. Li, J.; Chan, J.S.; Chow, A.Y.; Yuen, L.P.; Chan, C.L. From body to mind and spirit: Qigong exercise for bereaved persons with chronic fatigue syndrome-like illness. Evid. Based Complementary Altern. Med. 2015, 2015, 1-7. [CrossRef]

72. Wong, W.S.; Fielding, R. Construct validity of the Chinese version of the Chalder Fatigue Scale in a Chinese community sample. J. Psychosom. Res. 2010, 68, 89-93. [CrossRef] [PubMed]

73. Leung, C.; Wing, Y.; Kwong, P.; Shum, A.L.K. Validation of the Chinese-Cantonese version of the Hospital Anxiety and Depression Scale and comparison with the Hamilton Rating Scale of Depression. Acta Psychiatr. Scand. 1999, 100, 456-461. [CrossRef]

74. Ware, J.E., Jr.; Kosinski, M.; Keller, S.D. A 12-Item Short-Form Health Survey: Construction of scales and preliminary tests of reliability and validity. Med. Care 1996, 34, 220-233. [CrossRef]

75. Lam, C.L.; Eileen, Y.; Gandek, B. Is the standard SF-12 health survey valid and equivalent for a Chinese population? Qual. Life Res. 2005, 14, 539-547. [CrossRef] [PubMed]

76. Ng, S.; Yau, J.K.; Chan, C.L.; Chan, C.H.; Ho, D.Y. The measurement of body-mind-spirit well-being: Toward multidimensionality and transcultural applicability. Soc. Work Health Care 2005, 41, 33-52. [CrossRef]

77. Mundt, J.C.; Marks, I.M.; Shear, M.K.; Greist, J.M. The Work and Social Adjustment Scale: A simple measure of impairment in functioning. Br. J. Psychiatry 2002, 180, 461-464. [CrossRef]

78. Stewart, A.L.; Hays, R.D.; Ware, J.E. The MOS short-form general health survey: Reliability and validity in a patient population. Med. Care 1988, 26, 724-735. [CrossRef]

79. McHorney, C.A.; Ware, J.E., Jr.; Lu, J.R.; Sherbourne, C.D. The MOS 36-item Short-Form Health Survey (SF-36): III. Tests of data quality, scaling assumptions, and reliability across diverse patient groups. Med. Care 1994, 32, 40-66. [CrossRef]

80. Rimes, K.A.; Chalder, T. The Beliefs about Emotions Scale: Validity, reliability and sensitivity to change. J. Psychosom. Res. 2010, 68, 285-292. [CrossRef]

81. Neff, K.D. The development and validation of a scale to measure self-compassion. Self Identity 2003, 2, 223-250. [CrossRef]

82. Baer, R.A.; Smith, G.T.; Hopkins, J.; Krietemeyer, J.; Toney, L. Using self-report assessment methods to explore facets of mindfulness. Assessment 2006, 13, 27-45. [CrossRef]

83. Chan, J.S.; Li, A.; Ng, S.-M.; Ho, R.T.; Xu, A.; Yao, T.-J.; Wang, X.M.; So, K.F.; Chan, C.L. Adiponectin potentially contributes to the antidepressive effects of Baduanjin Qigong exercise in women with chronic fatigue syndrome-like illness. Cell Transpl. 2017, 26, 493-501. [CrossRef]

84. Buysse, D.J.; Reynolds, C.F., III; Monk, T.H.; Berman, S.R.; Kupfer, D.J. The Pittsburgh Sleep Quality Index: A new instrument for psychiatric practice and research. Psychiatry Res. 1989, 28, 193-213. [CrossRef]

85. Chong, A.M.; Cheung, C.-K. Factor structure of a Cantonese-version pittsburgh sleep quality index. Sleep Biol. Rhythms. 2012, 10, 118-125. [CrossRef]

86. Ho, R.T.; Fong, T.C. Factor structure of the Chinese version of the Pittsburgh Sleep Quality Index in breast cancer patients. Sleep Med. 2014, 15, 565-569. [CrossRef]

87. Fukuhara, S.; Suzukamo, Y. Manual of the SF-8 Japanese version. Kyoto: Institute for Health Outcomes \& Process Evaluation Research. Environ. Health Prev. Med. 2011, 16, 97-105.

88. Carruthers, B.M.; Jain, A.K.; De Meirleir, K.L.; Peterson, D.L.; Klimas, N.G.; Lerner, A.M.; Bested, A.C.; Flor-Henry, P.; Joshi, P.; Powles, A.P.; et al. Myalgic Encephalomyelitis/Chronic Fatigue Syndrome. J. Chronic Fatigue Syndr. 2003, 11, 7-115. [CrossRef]

89. Nolen-Hoeksema, S.; Morrow, J. A prospective study of depression and posttraumatic stress symptoms after a natural disaster: The 1989 Loma Prieta Earthquake. J. Personal. Soc. Psychol. 1991, 61, 115. [CrossRef]

90. Diener, E.; Emmons, R.A.; Larsen, R.J.; Griffin, S. The life satisfaction scale. J. Personal. Assess. 1985, 49, 71-75. [CrossRef] [PubMed] 
91. Oka, T.; Tanahashi, T.; Lkhagvasuren, B.; Yamada, Y. The longitudinal effects of seated isometric yoga on blood biomarkers, autonomic functions, and psychological parameters of patients with chronic fatigue syndrome: A pilot study. BioPsychoSocial Med. 2019, 13, 1-13. [CrossRef] [PubMed]

92. Carruthers, B.M.; van de Sande, M.I.; De Meirleir, K.L.; Klimas, N.G.; Broderick, G.; Mitchell, T.; Staines, D.; Powles, A.P.; Speight, N.; Vallings, R.; et al. Myalgic encephalomyelitis: International Consensus Criteria. J. Intern. Med. 2011, 270, 327-338. [CrossRef] [PubMed]

93. Komaki, G. The reliability and factorial validity of the Japanese version of the 20-item Toronto Alexithymia Scale. J. Psychosom. Res. 2003, 55, 143. [CrossRef]

94. Tait, R.C.; Pollard, C.A.; Margolis, R.B.; Duckro, P.N.; Krause, S.J. The Pain Disability Index: Psychometric and validity data. Arch Phys. Med. Rehabil. 1987, 68, 438-441.

95. Wicksell, R.K.; Renöfält, J.; Olsson, G.L.; Bond, F.W.; Melin, L. Avoidance and cognitive fusion-central components in pain related disability? Development and preliminary validation of the Psychological Inflexibility in Pain Scale (PIPS). Eur. J. Pain 2008, 12, 491-500. [CrossRef] [PubMed]

96. Smets, E.; Garssen, B.; Bonke, B.; De Haes, J. The Multidimensional Fatigue Inventory (MFI) psychometric qualities of an instrument to assess fatigue. J. Psychosom. Res. 1995, 39, 315-325. [CrossRef]

97. Rabin, R.; Charro, F.D. EQ-SD: A measure of health status from the EuroQol Group. Ann. Med. 2001, 33, 337-343. [CrossRef]

98. Tanaka, M.; Fukuda, S.; Mizuno, K.; Imai-Matsumura, K.; Jodoi, T.; Kawatani, J.; Takano, M.; Miike, T.; Tomoda, A.; Watanabe, Y. Reliability and validity of the Japanese version of the Chalder Fatigue Scale among youth in Japan. Psychol. Rep. 2008, 103, 682-690. [CrossRef]

99. Jackson, C. The Chalder fatigue scale (CFQ 11). Occup. Med. 2015, 65, 86. [CrossRef] [PubMed]

100. Hornig, M.; Montoya, J.G.; Klimas, N.G.; Levine, S.; Felsenstein, D.; Bateman, L.; Peterson, D.L.; Gottschalk, C.G.; Schultz, A.F.; Che, X.; et al. Distinct plasma immune signatures in ME/CFS are present early in the course of illness. Sci. Adv. 2015, 1. [CrossRef] [PubMed]

101. Landi, A.; Broadhurst, D.; Vernon, S.D.; Tyrrell, D.L.J.; Houghton, M. Reductions in circulating levels of IL-16, IL-7 and VEGF-A in myalgic encephalomyelitis/chronic fatigue syndrome. Cytokine 2016, 78, 27-36. [CrossRef]

102. Maes, M.; Mihaylova, I.; Kubera, M.; Leunis, J.C.; Twisk, F.N.M.; Geffard, M. IgM-mediated autoimmune responses directed against anchorage epitopes are greater in Myalgic Encephalomyelitis/Chronic Fatigue Syndrome (ME/CFS) than in major depression. Metab. Brain Dis. 2012, 27, 415-423. [CrossRef]

103. Strayer, D.R.; Victoria, S.; William, C. Low NK Cell Activity in Chronic Fatigue Syndrome (CFS) and Relationship to Symptom Severity. J. Clin. Cell. Immunol. 2015, 1. [CrossRef]

104. Papadopoulos, A.S.; Cleare, A.J. Hypothalamic-pituitary-adrenal axis dysfunction in chronic fatigue syndrome. Nat. Rev. Endocrinol. 2011, 8. [CrossRef] [PubMed]

105. Gupta, A. Unconscious amygdalar fear conditioning in a subset of chronic fatigue syndrome patients. Med. Hypotheses 2002, 59, 727-735. [CrossRef]

106. Dowsett, E.; Goudsmit, E.; Macintyre, A.; Shepherd, C. London criteria for ME. In Report from the National Task Force on Chronic Fatigue Syndrome (CFS), Post Viral Fatigue Syndrome (PVFS), Myalgic Encephalo-myelitis (ME); An Initiative of the Registered Charity Westcare; Department of Health and with Financial Assistance from the Wellcome Trust: Westcare, UK, 1994; pp. 96-98.

107. Jason, L.; Evans, M.; Porter, N.; Brown, M.; Brown, A.; Hunnell, J.; Biotechnol, A.J. The development of a revised Canadian myalgic encephalomyelitis chronic fatigue syndrome case definition. Am. J. Biochem. Biotechnol. 2010, 6, 120-135. [CrossRef] 